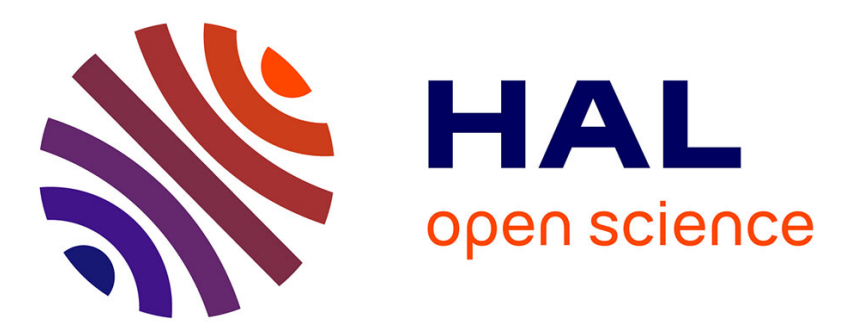

\title{
ERT monitoring of gas injection into water saturated sands: Modelling and inversion of cross-hole laboratory data
}

\author{
Thomas Kremer, Cristian Vieira, Alexis Maineult
}

\section{To cite this version:}

Thomas Kremer, Cristian Vieira, Alexis Maineult. ERT monitoring of gas injection into water saturated sands: Modelling and inversion of cross-hole laboratory data. Journal of Applied Geophysics, 2018, 158 (Novembre 2018), pp.11-28. 10.1016/j.jappgeo.2018.06.001 . hal-02140621

\section{HAL Id: hal-02140621 \\ https://hal.sorbonne-universite.fr/hal-02140621}

Submitted on 27 May 2019

HAL is a multi-disciplinary open access archive for the deposit and dissemination of scientific research documents, whether they are published or not. The documents may come from teaching and research institutions in France or abroad, or from public or private research centers.
L'archive ouverte pluridisciplinaire HAL, est destinée au dépôt et à la diffusion de documents scientifiques de niveau recherche, publiés ou non, émanant des établissements d'enseignement et de recherche français ou étrangers, des laboratoires publics ou privés. 
1 ERT monitoring of gas injection into water saturated sands: modelling and 2 inversion of cross-hole laboratory data.

4 Thomas Kremer $^{1,2, *}$, Cristian Vieira ${ }^{1}$ and Alexis Maineult ${ }^{1,2,3}$

5

6 1) Institut de Physique du Globe de Paris, Sorbonne Paris Cité, Univ. Paris Diderot. Paris, $7 \quad$ France.

8 2) Centre de recherches sur le stockage géologique du $\mathrm{CO}_{2}$ (IPGP / TOTAL / 9 SCHLUMBERGER / ADEME), Paris, France.

10 3) Sorbonne Université, CNRS, EPHE, UMR 7619 METIS, 4 place Jussieu, 75005 Paris, 11 France.

14 Keywords: $\mathrm{CO}_{2}$ and $\mathrm{N}_{2}$ injection; electrical resistivity tomography; saturated sands; transport modeling; cellular automaton; electrode configuration.

*Corresponding author. Now at University of Liege, Argenco Department, Applied

20 Geophysics, Allée de la découverte, 9, 4000, Liege, Belgium. E-mail:

21 kremer.tho@gmail.com. 
ABSTRACT

A set of laboratory experiments were performed in order to investigate the capacity of the electrical resistivity tomography method to detect and monitor the circulation of gaseous $\mathrm{CO}_{2}$ into geological media. The experiments consisted of injecting reactive $\left(\mathrm{CO}_{2}\right)$ or inert $\left(\mathrm{N}_{2}\right)$ gases into reactive (carbonate) or inert (silica) sands saturated with water. The laboratory setup was a metric-scale cylindrical tank instrumented with electrodes placed on vertical rods, hence simulating a borehole to borehole monitoring network. The results demonstrated that the ERT method can efficiently detect, locate and image gas circulation plumes. Two main behaviors were identified: either an increase of the medium resistivity due to the invasion of the porous space by an electrically insulating gas phase, or a resistivity decrease induced by dissolution processes that enhance ionic concentrations in the saturating water. The observed anomalies showed varying geometric shapes depending on which type of sand was used. A 3D cellular automaton model was built and managed to reproduce different gas plumes circulation patterns and the electrical resistivity response associated. This modeling work confirmed the link existing between the shapes of the resistivity anomalies, the gas plumes geometry and the particle size distribution of the sand. Through a numerical sensitivity analysis, several measuring protocols were tested and developed in order to improve the imaging capacity of the electrical resistivity method. Results demonstrated that the most efficient protocol was different depending on the shape of the gas plume, thus stressing the importance of having a priori knowledge on the geological, lithological and hydraulic characteristics of the subsurface, in order to correctly assess the most likely gas plume geometry and hence design the most adapted monitoring protocol. 


\section{INTRODUCTION}

Since the beginning of the industrial era, anthropogenic activities such as combustion

of fossil resources and deforestation have resulted in a continuous increase of atmospheric $\mathrm{CO}_{2}$ concentrations (Neftel et al., 1985; Keeling et al., 2009), which have recently passed over the symbolic threshold of $400 \mathrm{ppm}$ (Showstack, 2013). It is very likely that these elevated concentrations participate to major environmental impacts such as climate change (Manabe and Stouffer, 1980; Cox et al., 2000) and ocean acidification (Doney et al., 2009). Carbon capture and storage (CCS), which consists of capturing the $\mathrm{CO}_{2}$ emitted by high emissions industrial units and storing it in deep geological formations, is considered today as a viable strategy to mitigate these impacts.

To ensure the long term efficiency of the process and prevent environmental impacts associated with $\mathrm{CO}_{2}$ leakage into nearby aquifers, it is vital to develop and utilize monitoring tools that allow the detection and quantification of any $\mathrm{CO}_{2}$ transfer out of the targeted geological formation. Several approaches can be employed to achieve this goal. Some of them focus on the monitoring of the $\mathrm{CO}_{2}$ plume itself (generally in the supercritical state), and aim to detect any change occurring in this area. Other strategies are directed toward the monitoring of the geological formations located above the plume up to the surface, in which case the goal is to detect a prospective $\mathrm{CO}_{2}$ transfer within these structures (which would likely happen under the gaseous state if the pressure and temperature conditions allow it). The present study falls in the second category. Several approaches can be employed; in particular, geophysical methods can be used to study in an indirect way various physical properties of the subsurface, and detect possible variations of these properties due to the presence of leaking $\mathrm{CO}_{2}$. Among them, the electrical resistivity tomography (ERT) method is of particular interest because the electrical resistivity of geologic materials is sensitive to the nature, the 
composition and the distribution of fluids in the porous space. Furthermore, the costs of implementation are significantly lower than those of seismic methods (generally used by the oil and gas industry), thus providing a monitoring strategy with a higher temporal discretization.

To date, the ERT method has been successfully applied by numerous authors for $\mathrm{CO}_{2}$ detection into water saturated geological media (Xue et al., 2006; Bergmann et al., 2017; Auken et al., 2014). Depending on the physico-chemical state of the studied medium, two main processes can affect the ERT response of the ground. In some cases, the gaseous $\mathrm{CO}_{2}$ is observed to cause a global increase of the medium resistivity, as it acts as an electrically insulating phase within the porous space. Such effects have been observed and well documented through field experiments (Xue et al., 2006; Kiessling et al., 2010; Bergmann et al., 2017; Carrigan et al., 2013) and at the laboratory scale (Nakatsuka et al., 2010; Breen et al., 2012; Bosch et al., 2016). In other cases, it has been observed that the bulk resistivity of the ground can decrease when important dissolution processes are occurring (Peter et al., 2012; Vialle et al., 2014; Auken et al., 2014). The general sequence of events starts with the dissolution of some of the gaseous $\mathrm{CO}_{2}$ into the saturating water, which becomes more acidic, thus enhancing the potential dissolution of minerals. All the newly dissolved ionic species lead to a more electrically conductive water and hence to a less resistive medium. In some cases, dissolution and desaturation effects can be observed simultaneously (Xue et al., 2006; Barrio et al., 2014, Yang et al., 2015).

In this paper, we present metric scale experiments which aim to simulate a gaseous $\mathrm{CO}_{2}$ leakage through a relatively simple medium (well characterized sand saturated with water) and monitor the resistivity variations induced by the gas circulation. Although strong differences obviously exist, these experiments can be seen as an attempt to approach the conditions of a borehole-to-borehole ERT monitoring network through which a $\mathrm{CO}_{2}$ leakage 
100 path would form, in geological conditions close to those of a saturated aquifer with a

101 relatively homogeneous structure. Two different sands were used in order to study in parallel

102 carbonated environments (in which strong dissolution processes can occur) and silica based

103 environments (in which dissolution processes are very weak). The experiments were

104 performed using gaseous $\mathrm{CO}_{2}$ and gaseous $\mathrm{N}_{2}$ in order to study separately the "insulating

105 phase" effect, when gas invades the porous space and no dissolution processes occur. Before

106 the experiments, a preliminary study was conducted to investigate different ERT measuring

107 protocols and choose an optimal one. Then, we present the results of the laboratory

108 experiments. In view of the results, a model was designed under the form of a cellular

109 automaton with stochastic behavior, in order to simulate gas injection and circulation through

110 the medium. Coupling this model with the software R3t (Binley 2013), we were able to

111 reproduce the ERT responses observed during the experiments and to infer the geometry of

112 the gas plume, which changes depending on the grain size distribution of the sand. Finally, in

113 a third phase, the cellular automaton model was used as a tool to study numerically the

114 sensitivity of the electrodes configurations and to develop new, more efficient acquisition 115 protocols.

\section{MATERIALS AND METHODS}

\subsection{Experimental set-up}

To perform the experiments, an 80-cm-high, 48-cm-diameter cylindrical Plexiglas tank

120 was used (Fig. 1a). Electrodes were arranged in 10 rings of 6 cylindrical electrodes fixed on

121 plastic rods attached on the inner wall of the tank. The vertical ring spacing was $5 \mathrm{~cm}$, and the

122 lowest ring was located $18 \mathrm{~cm}$ above the bottom of the tank (Fig. 1b). The electrodes were not

123 placed exactly on the wall of the tank due to the diameter of the rods $(1 \mathrm{~cm})$ and the space

124 between each rod and the internal wall $(3 \mathrm{~mm})$. The electrodes $(4 \mathrm{~mm}$ in diameter and $5 \mathrm{~mm}$ 
125 in length) were made of stainless steel to prevent from corrosion. Gas injection was performed 126 using a valve placed at the bottom center of the tank. The gas was injected through a 127 cylindrical air diffuser $(10 \mathrm{~cm}$ in height and $12 \mathrm{~cm}$ in diameter, Fig. 1c) placed on the axis of

128 the tank, between the planes 10 and 8. A small pipe connects the diffuser to a valve placed at 129 the bottom center of the tank, and linked to gas reserves. All these components were made of $130 \quad$ PVC to minimize electrical disturbances.

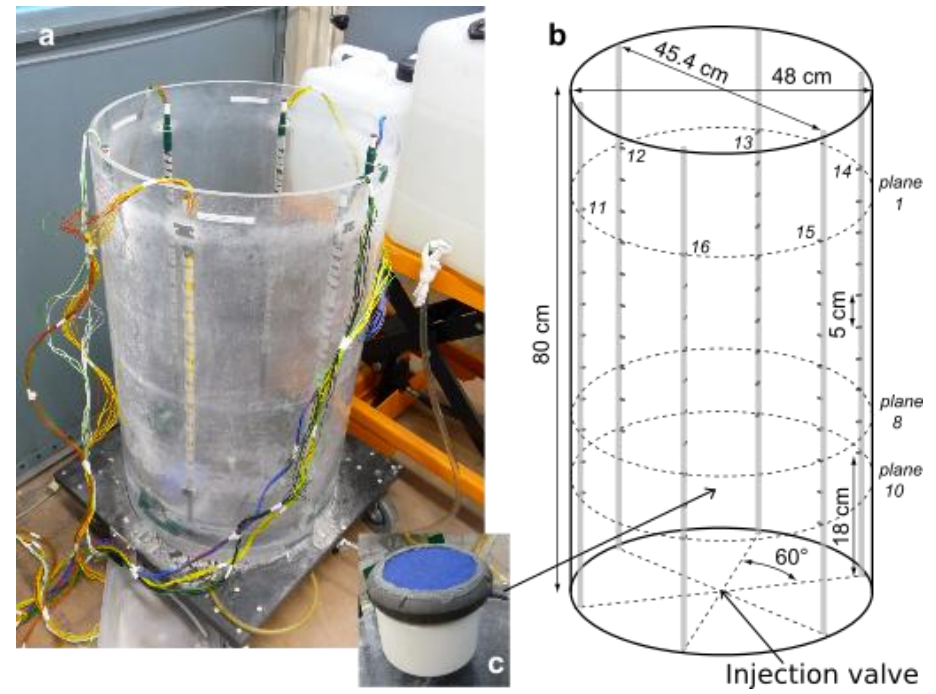

Fig. 1. Experimental set-up. Picture of the cylindrical tank equipped with the electrodes (a) and of the gas diffuser (c). Sketch of the tank and position of the electrodes (b).

\subsection{Porous medium}

145 Fontainebleau (France) and the second is a carbonate sand obtained from commercial

146 aggregates. The porosity values of the sands were estimated using decimetric columns filled

147 with sand, by measuring the mass difference between the unsaturated state and the water

148 saturated state. The permeability estimates were obtained by applying different hydraulic

149 heads values on the saturated columns and monitoring the hydraulic flow. The structural and 
hydraulic characteristics of the sands are listed in Table 1, where errors were calculated as the

151 standard deviation of the results obtained on 4 independent measurements.

Table 1. Characteristics of the sands

\begin{tabular}{|l|c|c|}
\cline { 2 - 3 } \multicolumn{1}{c|}{} & \multicolumn{2}{c|}{ Sand } \\
\cline { 2 - 3 } \multicolumn{1}{c|}{} & Silica & Carbonate \\
\hline Composition & $98 \%$ mass silica & $98 \%$ molal $\mathrm{CaCO}_{3}$ \\
& $2 \%$ mass feldspars & $2 \%$ molal $\mathrm{MgCO}_{3}$ \\
\hline Grain size & $200-400 \mu \mathrm{m}$ & $50-2000 \mu \mathrm{m}$ \\
\hline Porosity $(\%)$ & $39 \pm 1$ & $45 \pm 1$ \\
\hline Permeability $\left(\mu \mathrm{m}^{2}\right)$ & $4.5 \pm 0.5$ & $16.5 \pm 1.3$ \\
\hline
\end{tabular}

To create a homogeneous distribution in the tank and avoid bubble trapping, the sand was slowly, regularly poured into water using a sieve whose mesh size is slightly larger than the largest grain size. Sand and water were then added and stirred simultaneously so as to always keep a free water layer of a few centimeters thick at the top of the medium, which was removed once the filling procedure was completed. Such procedure ensured that no significant air bubbles remained compared to the volume of the tank and the amount of gas injected during the experiments. The air diffuser was placed after the lowest ring of electrodes was reached. We avoided the formation of unfilled cavities in the zones between the vertical plastic rods and the internal wall of the tank, since they could act as preferential gas migration

164 pathways. No compaction process was applied during the filling procedure except in these 165 zones.

\subsection{Gas injection and dissolution processes}

We employed dinitrogen $\left(\mathrm{N}_{2}\right)$ and carbon dioxide $\left(\mathrm{CO}_{2}\right)$ during the experiments. temperature $\left.\mathrm{T}=0^{\circ} \mathrm{C}\right)$, and does not react with the solid phase, while carbon dioxide $\left(\mathrm{CO}_{2}\right)$ 
171 has a solubility into water at $0^{\circ} \mathrm{C}$ of about 0.88 and can react with the solid matrix once

172 dissolved in water. In the conditions of the experiments, these gases can be considered as 173 perfect insulators (their resistivity is about 15 orders of magnitude higher than the resistivity

174 of the water used during the experiments). It is important to note that we worked with an 175 opened system, where the injected gas is allowed to pass through and flow out of the medium 176 (the tank is not covered up). Gas injection is done according to the following protocol: a first 177 injection phase is performed at a rate of about $200 \mathrm{~L} \cdot \mathrm{h}^{-1}$ for at least $30 \mathrm{~min}$, creating a steady state with multiple gas pockets spread in the tank and gas migration pathways that seem stable, as indicated by the observation of bubbles appearing continuously at the same

180 locations at the surface of the medium. Then, the injection rate is increased to $400 \mathrm{~L} \cdot \mathrm{h}^{-1}$. Once 181 a steady-state is reached (about 30 min later) the ERT acquisition starts and the injection is 182 sustained until the whole acquisition sequence is performed, which lasted about 1.5 hours. Experiments involving $\mathrm{N}_{2}$ injection allowed studying the case where changes of resistivity are solely induced by an increase of the gas saturation, and where no dissolution processes occur. On the other hand, during $\mathrm{CO}_{2}$ injection, chemical reactions due to carbonic acid might occur depending upon mineralogy (Xu et al., 2005; Busch et al., 2009; Raistrick et al., 2009). When put in contact with water, part of the gaseous $\mathrm{CO}_{2}$ dissolves and reacts with water forming carbonic acid, which will itself dissociates, providing additional ionic species into the water. Those processes can be summarized by the following equations.

190

$$
\mathrm{CO}_{2(\mathrm{~g})} \leftrightarrows \mathrm{CO}_{2}(\mathrm{aq})
$$

$$
\mathrm{CO}_{2(\mathrm{aq})}+\mathrm{H}_{2} \mathrm{O}_{(\mathrm{aq})} \leftrightarrows \mathrm{H}_{2} \mathrm{CO}_{3}(\mathrm{aq})
$$

$$
\mathrm{H}_{2} \mathrm{CO}_{3(\mathrm{aq})}+\mathrm{H}_{2} \mathrm{O}_{(\mathrm{aq})} \leftrightarrows \mathrm{HCO}_{3}^{-}+\mathrm{H}_{3} \mathrm{O}^{+}
$$

$$
\mathrm{HCO}_{3}^{-}+\mathrm{H}_{2} \mathrm{O}_{(\mathrm{aq})} \leftrightarrows \mathrm{CO}_{3}^{2-}+\mathrm{H}_{3} \mathrm{O}^{+}
$$


In the case of a solid phase composed of calcium carbonate, the dissolved $\mathrm{CO}_{2}$ will also enhance the dissolution of part of the solid matrix, further increasing the ionic concentrations in the saturating water.

199

$$
\mathrm{CaCO}_{3}+\mathrm{CO}_{2}(\mathrm{aq})+\mathrm{H}_{2} \mathrm{O}_{(\mathrm{aq})} \leftrightarrows \mathrm{Ca}^{2+}+2 \mathrm{HCO}_{3}^{-}
$$

$$
\text { modify the water resistivity value. Therefore, in experiments involving } \mathrm{CO}_{2} \text { injection, two }
$$
phenomena can possibly affect the effective resistivity of the media: 1) water saturation level variation due to gas invasion, which would provoke an increase of the effective media resistivity and 2) dissolution processes, which would result in a decrease of the media resistivity. In the following, we will refer to these two phenomena respectively as the "insulating phase" effect and the "dissolution processes" effect.

\subsection{Data acquisition and processing}

The acquisition device was a Terrameter SAS 1000 connected with a 64-electrode multiplexer ES-1064 by ABEM. Injected current consists of square waves of alternating polarity of $20 \mathrm{~mA}$ to avoid polarization and associated noise (Parasnis, 1997). The acquisition delay was set to $0.1 \mathrm{~s}$ and the acquisition time to $0.2 \mathrm{~s}$.

To perform a tomography reconstruction, we measured the potential differences resulting from current injection for a large set of electrodes arrays. Finally, to obtain an estimation of the resistivity distributions from the measured resistances, inversions were carried out with the software R3t (Binley, 2013), as it is suitable to the medium geometry and experimental conditions. R3t is based on a regularized objective function with weighted least square. Neumann conditions are specified at the boundaries where no current flow is allowed 
222 Gauss-Newton procedure, as described in Binley \& Kemna (2005). In this study we used the 223 background regularization scheme described in Binley (2013), where the regularization 224 parameter is optimized each iteration by carrying out a line search. The convergence of the 225 inversion process occurred only when the root-mean-square (RMS) error, as defined in Binley 226 (2013), was below 1.

The electrodes were modeled as point sources. This was considered an acceptable hypothesis given the results from Rücker \& Günther (2011). According to their study, the errors related to the point source assumption become important when the ratio between the electrode spacing and electrode length overcomes 0.2. In the present study this ratio never overcomes 0.1 for all measurements.

As explained in Binley et al., (1995, 1996a, 1996b), Binley \& Kemna (2005) and, 233 Daily et al., (2004), the characterization of the error is required for ERT modeling to assess its effect during reconstruction of the true resistivity distribution. The error can be inferred from repeatability and reciprocity. Repeatability is related to the precision at which measurements are obtained under identic conditions. Reciprocity is based on the principle that switching 237 positions between injection electrodes $\mathrm{A}$ and $\mathrm{B}$ and measuring electrodes $\mathrm{M}$ and $\mathrm{N}$ should 238 yield the same resistance value. The measurement error was estimated using both repeatability and reciprocity 240 principles (Binley \& Kemna, 2005 ; Daily et al., 2004) during a preliminary experiment $\left(\mathrm{N}_{2}\right.$ 241 injection into saturated siliceous sand). One estimation was made before starting the gas 242 injection and another one while gas injection was occurring but when gas migration pathways 243 seemed stable. The error inferred from reciprocity was found to be always less than $1 \%$ in 244 both cases. This value was considered low enough to be ignored and reciprocity was therefore 245 not assessed during the experiments presented in this paper. Only the repeatability error was 246 taken in account in the inversion process. 


\subsection{Optimal acquisition arrays}

An important issue in ERT surveys relates to the sensitivity of the electrode configurations used for the acquisition and, by extension, to the sensitivity of the measuring protocol. The optimization of the electrode configurations on the surface of the soil (surface ERT) has been studied by several authors (Dahlin \& Zhou, 2004; Christensen et al., 2006; Szalai et al., 2009). To our knowledge, most cross-well ERT monitoring strategies implement dipole-dipole or bipole-bipole arrays for acquisition (Pezard et al., 2016; Commer et al., 2016 ; Yang et al., 2015, Preignitz et al., 2013) sometimes mixing both arrays (Hermans et al., 2015). A review of pros and cons of some classical cross-borehole arrays are also presented in Bing \& Greenhalgh (2000). In the case of measurements at the surface of a cylinder, Binley \& Kemna (2005) recommend to use all linearly independent combinations. We chose to investigate this issue for our particular case, by testing several protocols on simple media with uniform resistivity distribution. The arrays bipole-bipole, dipole-dipole, Schlumberger and the protocol called cross-rods configuration (recommended by Binley \& Kemna, 2005) were tested. For the bipole-bipole array (Fig. 2a), current adjacent electrodes are located in the same plane whilst potential electrodes are located on the same position in the upper or lower plane. In dipole-dipole (Fig. 2b) the current electrodes are placed in the same rod and the potential electrodes in the adjacent rod, but the separation between $\mathrm{A}$ and $\mathrm{B}$ and $\mathrm{M}$ and $\mathrm{N}$ covered all possible combinations. In the Schlumberger array (Fig. 2c) all the electrodes were placed in the same rod where the distance between $\mathrm{A}$ and $\mathrm{M}$ equals the distance between $\mathrm{N}$ and B. The cross-rods configuration consists of all possible 4-electrodes combinations located 268 in the same plane (on the same ring), for each plane. All the possible 4-electrodes combinations are represented on Fig. 13. The main advantage of this protocol is that it forces the current lines to pass through the sand pack, enhancing the penetration depth. 
a

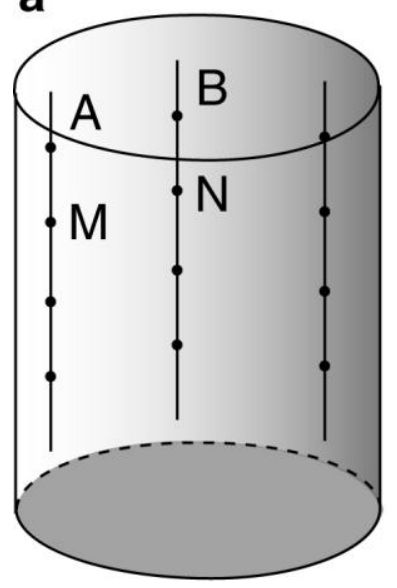

b

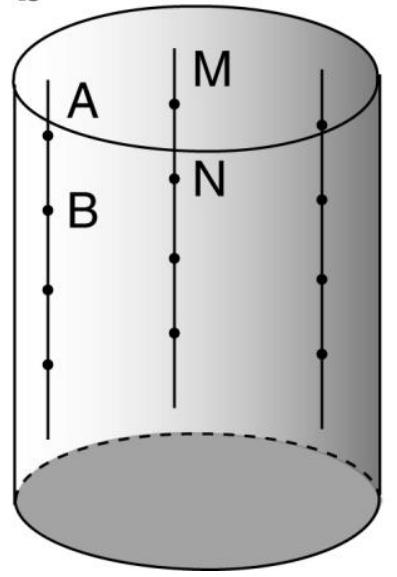

C

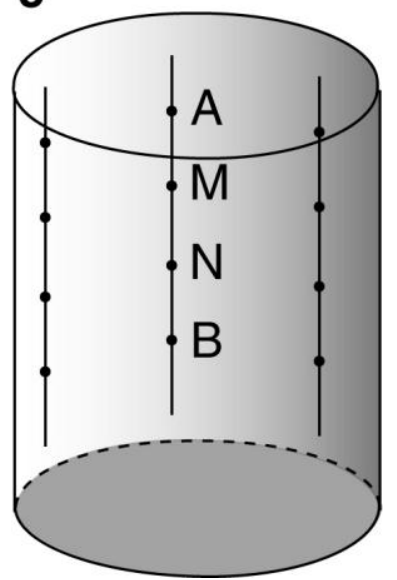

Fig. 2. Scheme of the "classical" acquisition arrays: bipole-bipole (a), dipole-dipole (b) and Schlumberger (c). Letters $A$ and $B$ denote the positions of the current injection electrodes and $M$ and $N$ that of the potential measurement electrodes.

As a first test, for each protocol, the resistances measured on a medium composed of

silica sand fully saturated with water were compared with the resistances values obtained through forward modelling of the same medium. The silica sand was saturated with a $\mathrm{NaCl}$ solution concentrated at 0.56 g.L $\mathrm{L}^{-1}$. The resistivity of such medium was measured thanks to a test cell, which yielded a value of $38 \Omega \cdot \mathrm{m}$. This resistivity value was then used to perform a forward modeling of the acquisition. Fig. 3 displays the results obtained with the different protocols tested. Concordance between measured and modeled resistances is much better when the cross-rods protocol is used than when the other "classical" configurations are used (bipole-bipole, dipole-dipole or Schlumberger). Given these results, we used exclusively the cross-rods protocol in the experiments described hereafter. 

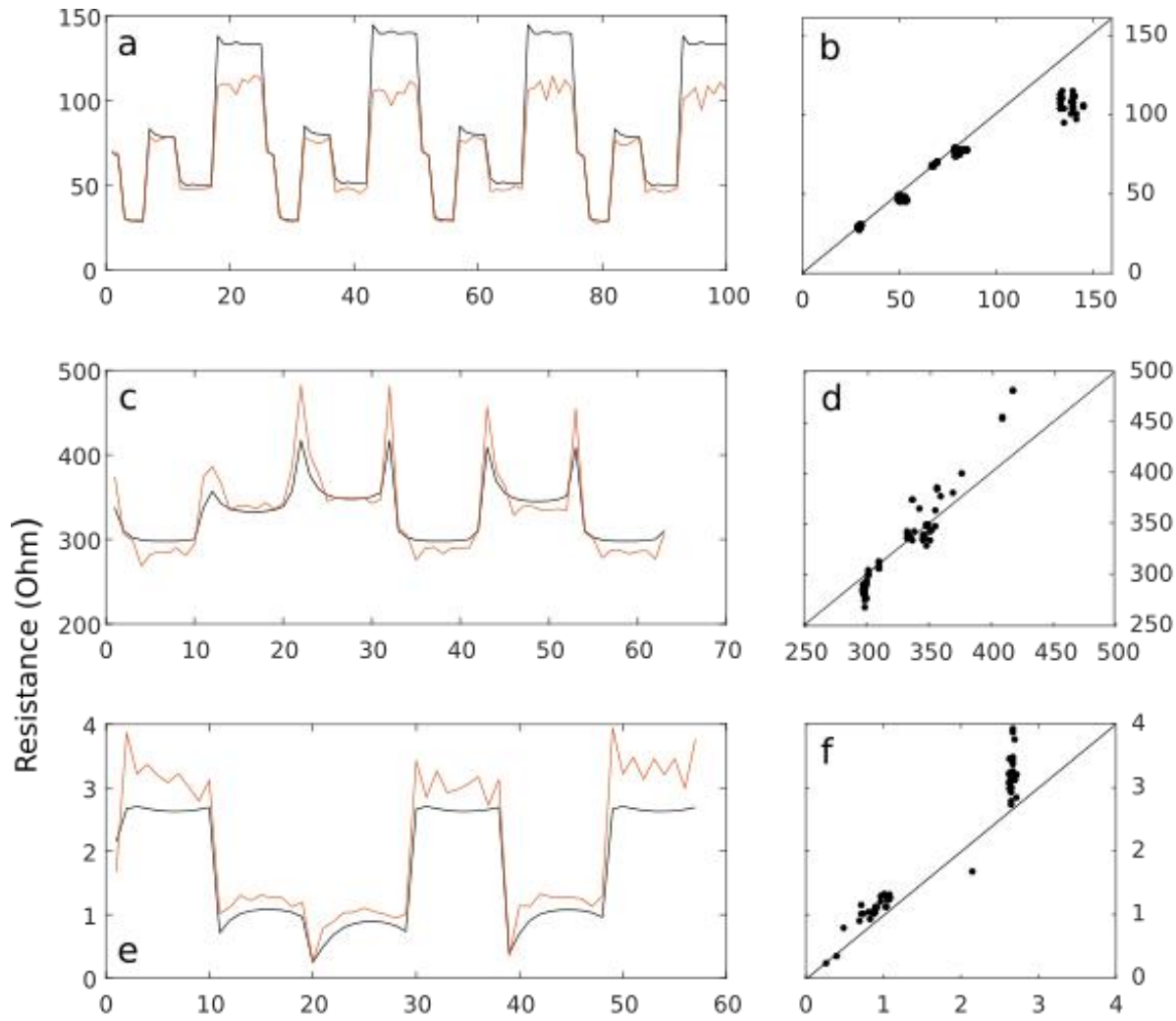

304
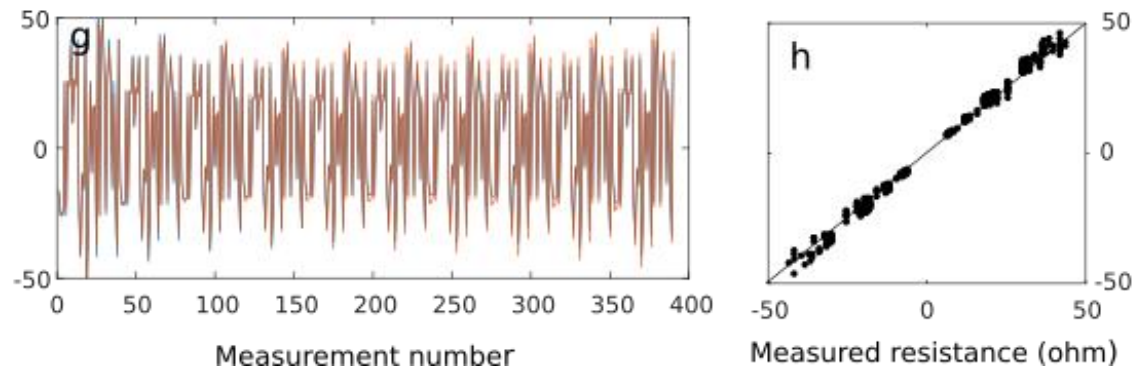

308

Fig. 3. Comparison between measured and modeled resistances. (a,b): Schlumberger protocol; (c,d): bipole-bipole; (e,f): dipole-dipole; (g,h): cross-rods configuration. Left column: measured (orange) and modeled (black) resistances values for each measurement. Right column: modeled vs measured resistances. 


\section{EXPERIMENTAL RESULTS}

As explained in the previous section, two types of gas $\left(\mathrm{N}_{2}\right.$ and $\left.\mathrm{CO}_{2}\right)$ and two types of sand (silica and carbonate) were used in this study, which resulted in the realization of four different experiments, one for each gas/sand combination. In the following, we will refer to each experiment using the initial of the sand type and the gas type formula. For example, $\mathrm{C} / \mathrm{N}_{2}$ will be referring to the experiment involving the injection of dinitrogen into saturated carbonate sand.

\subsection{ERT measurements on pure water}

Fig. 4 shows the resistivity distribution obtained after inversion when applying the $20^{\circ} \mathrm{C}$. The values obtained after inversion are very close to the true water resistivity value, indicating the good performance of the measuring protocol and the inversion process. Only few artifacts can be observed toward the lateral zones of the tank, mainly located in the more that $7 \%$ from the real water resistivity value.
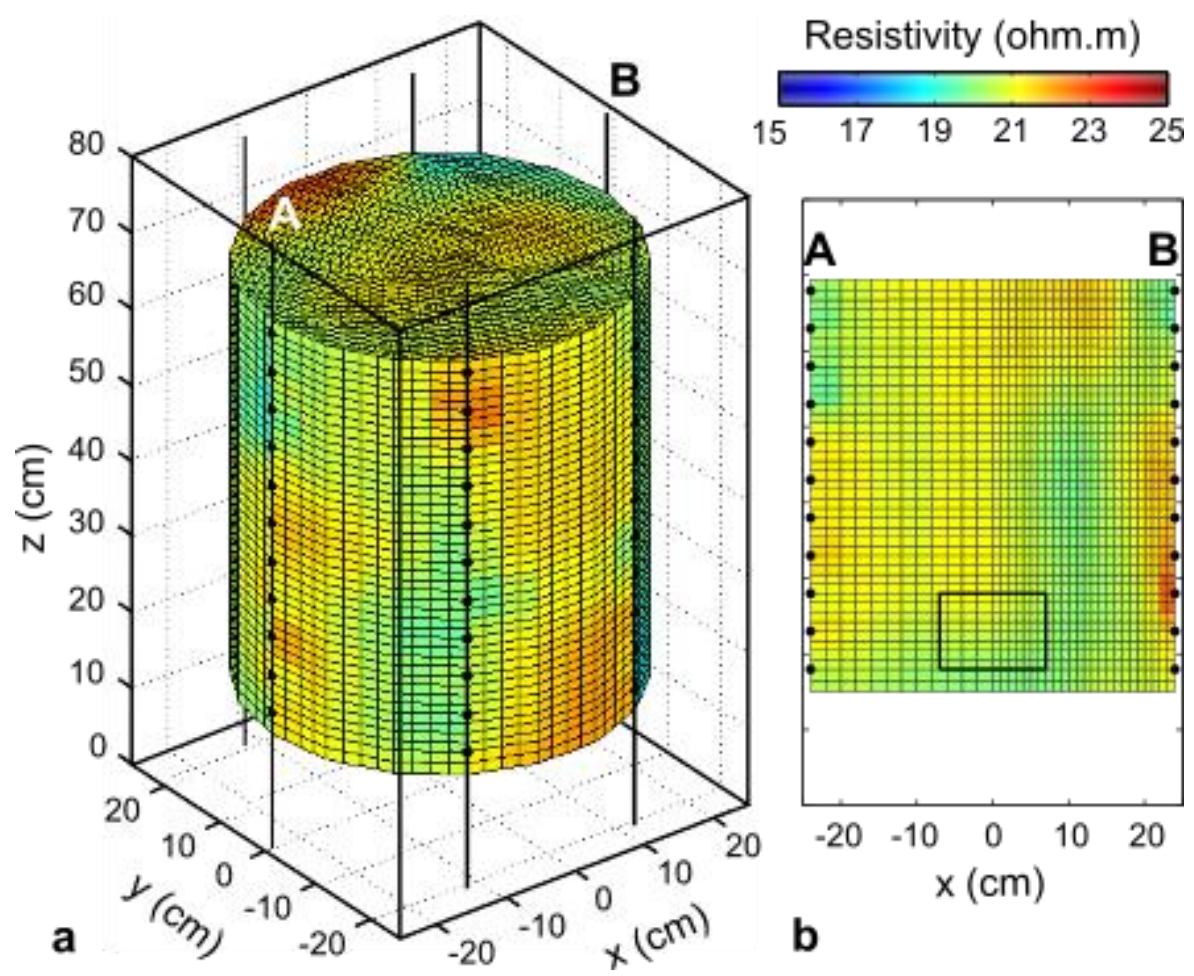

Fig. 4. Resistivity distribution obtained after inversion of ERT measurements performed on water only (water resistivity is $21.3 \Omega$.m). a) Isometric view of the 3D model. b) $2 \mathrm{D}$ section between rods denoted $A$ and $B$. The black square represents the gas diffuser position. 


\subsection{Gas injection into carbonate sand}

Fig. 5 shows the resistivity distribution obtained for the experiments involving carbonate sand $\left(\mathrm{C} / \mathrm{N}_{2}\right.$ and $\left.\mathrm{C} / \mathrm{CO}_{2}\right)$. For these experiments, the carbonate sand has been saturated with a $\mathrm{NaCl}$ solution whose initial concentration was 0.25 g.L $\mathrm{L}^{-1}(\rho=18.5 \Omega . \mathrm{m})$. During and after sand saturation, the resistivity of the water was found to decrease slowly, until reaching a stable value of about $14 \Omega \cdot \mathrm{m}$ at $17.5^{\circ} \mathrm{C}$, which is most likely due to the 348 dissolution of some carbonated particles during and immediately after the saturation process.

349 Fig. 5a shows the resistivity distribution of the initial state, before gas injection, which is quite 350 homogeneous except for some small artifacts in the vicinity of the electrodes. A slightly 351 higher resistivity zone can also be observed in the central part of the tank, which might be a 352 consequence of the plastic gas diffuser presence.

Fig. $5 \mathrm{~b}$ and $5 \mathrm{c}$ show the results of experiments $\mathrm{C} / \mathrm{N}_{2}$ and $\mathrm{C} / \mathrm{CO}_{2}$, respectively. For both experiments, the resistivity distributions obtained are no longer uniform. The $\mathrm{N}_{2}$ injection results in the formation of a high resistivity anomaly, likely to be induced by gas accumulation into the porous space. This anomaly exhibits a u-shape stretching from the gas

357 diffuser to the surface of the tank and represents a significant resistivity increase of about 50

$358 \%$ compared to the initial state. The injection of gaseous $\mathrm{CO}_{2}$ results, on the contrary, in a 359 decrease of the resistivity values compared to the initial state. This anomaly is also u-shaped, 360 centered with the axis of the tank, and stretching from the top of the diffuser to the surface of 361 the sand pack. It is likely that the injection of gaseous $\mathrm{CO}_{2}$ into a reactive medium such as 362 carbonate sand leads to strong dissolution processes which increase the electrical conductivity 363 of the saturating water and hence reduce the medium resistivity. In this case, even if gas 364 accumulation is also occurring, the impact of dissolution processes is stronger and dominates 365 the resistivity response of the medium. 
367 a rate of $200 \mathrm{~L} \cdot \mathrm{h}^{-1}$ followed by a second 30 min duration injection at a rate of $400 \mathrm{~L} \cdot \mathrm{h}^{-1}$. At

368 that point the "steady-state" has been reached (gas circulation patterns seem stable through

369 visual inspection of the surface of the medium) and the ERT acquisition starts and lasts for

370 about 1.5 hours. We note that the "steady-state" assumption is valid for experiment $\mathrm{C} / \mathrm{N}_{2}$ but

371 not necessarily for experiment $\mathrm{C} / \mathrm{CO}_{2}$, where dissolution processes might still be occurring

372 while the ERT acquisition is still in progress, thus leading to a kind of average resistivity

373 distribution over the duration of the ERT acquisition (even though most dissolution processes

374 are likely to occur before the beginning of ERT acquisition, given the fast kinetics of these

375 reactions).
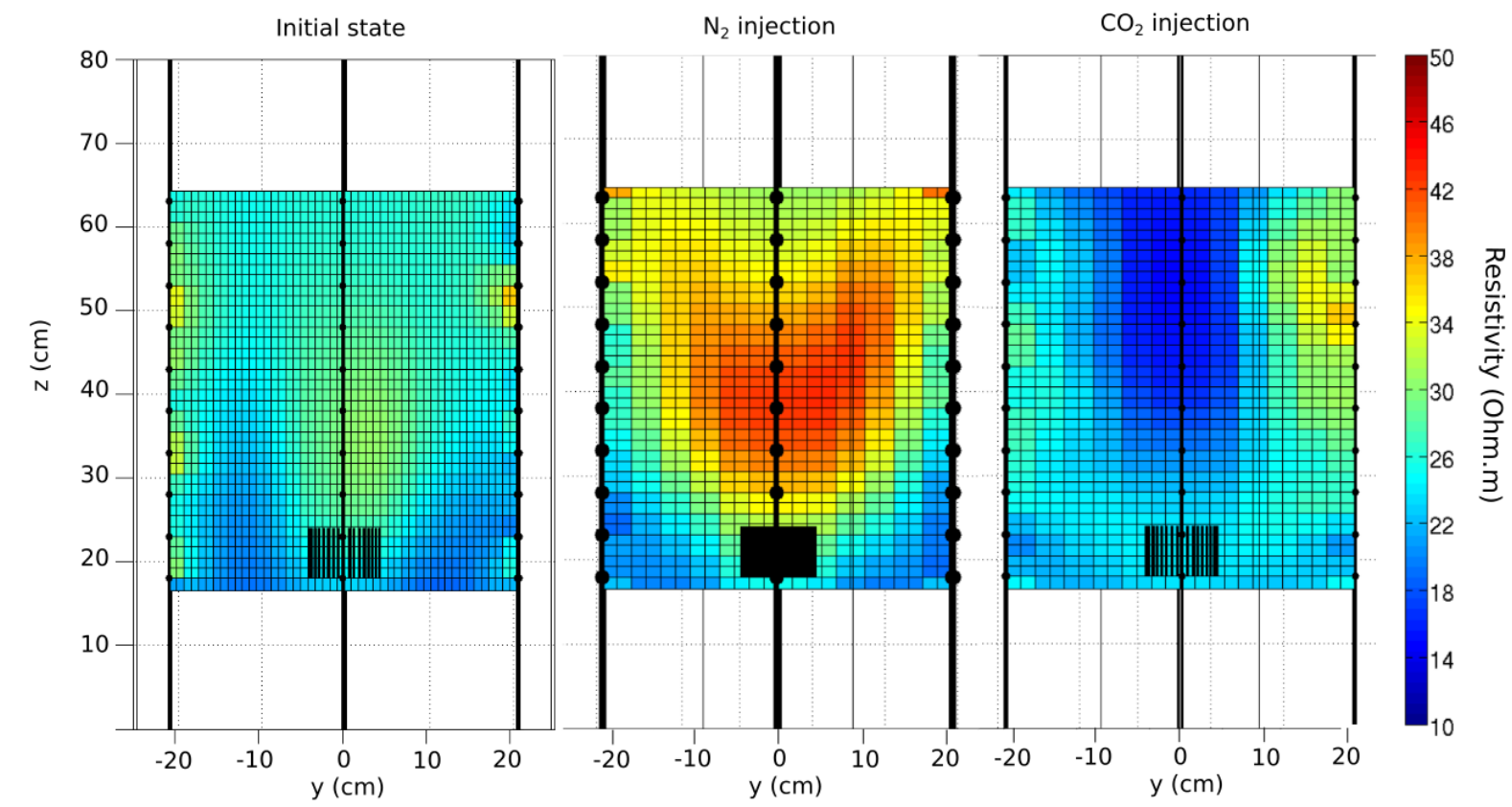

Fig. 5. Resistivity distribution obtained after inversion of ERT data acquired on carbonate sand saturated with water

of resistivity $14 \Omega . \mathrm{m}$ : at the initial state (left), during $\mathrm{N}_{2}$ injection of (middle), and during $\mathrm{CO}_{2}$ injection (right). The black rectangle indicates the position of the diffuser. 
391 injection only, and remove the effects induced by the presence of the gas diffuser. This 392 method is called the ratio-inversion. As explained in Miller et al., (2008) and Daily et al., 393 (2004), a common approach for detecting changes from a background image is to normalize 394 the data according to the following equation:

395

$$
d_{n}=\frac{d_{t}}{d_{0}}
$$
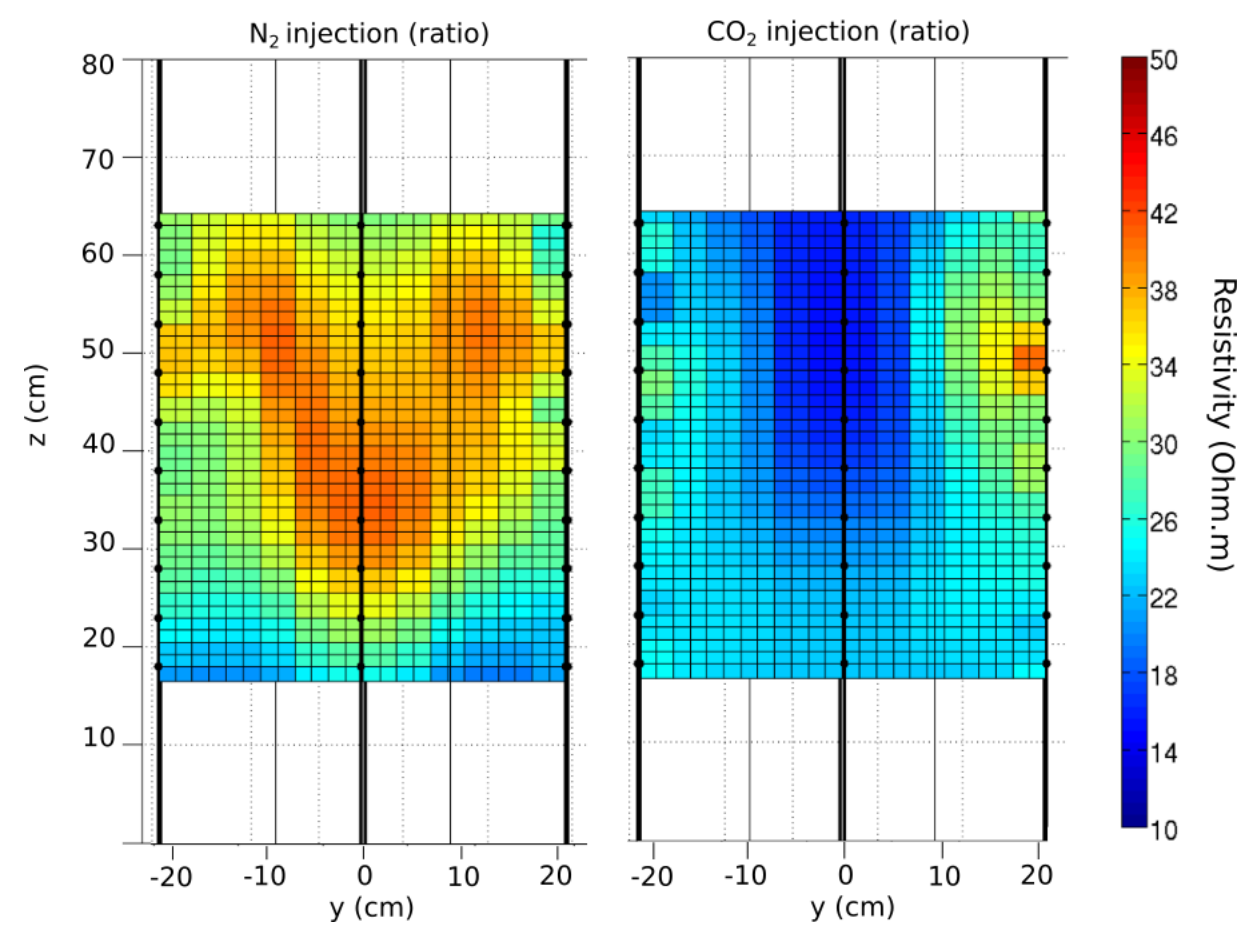

Fig. 6. Resistivity distribution obtained after application of ratio inversion on the data from experiments $\mathrm{C} / \mathrm{N}_{2}$ (left) and $\mathrm{C} / \mathrm{CO}_{2}$ (right). The resistivity anomalies remain u-shaped, stretching from the top of the diffuser to the top of the tank, and are centred with respect to the axis of the 
Fig. 6 show the results obtained with the ratio inversion method for experiment $\mathrm{C} / \mathrm{N}_{2}$

415 and $\mathrm{C} / \mathrm{CO}_{2}$. The shapes of the anomalies are globally very similar to those obtained with a 416 "simple" inversion, i.e u-shaped, centered with respect to the tank's vertical axis and 417 stretching from the top of the diffuser to the surface of the sand pack. Based on these results, 418 the ratio inversion method does not seem to provide significantly more accurate results, since 419 the same shapes can be observed with a simple inversion. However, if one would intent to use 420 these results in order to quantify the quantity of $\mathrm{N}_{2}$ or $\mathrm{CO}_{2}$ injected, it should be done with the 421 ratio inversion results, which are supposed to take in account only the resistivity structures 422 induced by gas injection, and exclude those present in the background resistivity distribution 423 (like the gas diffuser).

\subsection{Gas injection into silica sand}

Fig. 7 shows the resistivity distribution obtained for the experiments involving silica sand $\left(\mathrm{S} / \mathrm{N}_{2}\right.$ and $\left.\mathrm{S} / \mathrm{CO}_{2}\right)$. For these experiments, the sand was saturated with an electrolyte whose resistivity was $21 \Omega \cdot \mathrm{m}$ at $18.5^{\circ} \mathrm{C}$, which resulted in a higher background resistivity than for the carbonate experiments. As for the carbonate sand experiments, the initial state resistivity distribution (not shown here) was again quite homogeneous, exhibiting values mainly between 40 and $50 \Omega \cdot \mathrm{m}$ and only few artifacts in the vicinity of the electrodes. Fig $7 \mathrm{a}$ shows the resistivity results obtained with a simple inversion. The injection of gaseous $\mathrm{N}_{2}$ induces again the formation of a high resistivity anomaly, with values above $70 \Omega \cdot \mathrm{m}$, that we

434 can attribute to the invasion of the porous space by the insulating gaseous phase. However, the 435 shape of the anomaly is quite different since it does not seem to reach the upper part of the 436 tank, as if most of the gas was being trapped in the lower part. The ratio-inversion method 437 (Fig. 7b) manages to reduce some part of the anomaly that was likely due to the presence of 438 the diffuser, indicating that gas accumulation in the lowest part of the tank might not be as 
439 significant as the simple inversion results might suggest. However, the shape of the anomaly

440 remain mainly horizontal, not stretching toward the top of the sand pack and hence being

441 quite different from the results obtained with carbonate sand. Fig. 7c shows the results of the

442 ratio inversion for experiment $\mathrm{S} / \mathrm{CO}_{2}$. Again, we observe a high resistivity anomaly, with

443 values even higher than for experiment $\mathrm{S} / \mathrm{N}_{2}$. Even though it is likely that some of the $\mathrm{CO}_{2}$

444 has been dissolving into the water, the "insulating phase" effect seems to be dominating in

445 this case. In this case, the zone around the diffuser still exhibits high resistivity values after

446 ratio inversion, which might be attributed to significant gas accumulation around the diffuser

447 itself. Also, the anomaly again seems restricted to the lower part of the tank, showing

448 horizontal components rather than the u-shape observed with carbonate sand. These different

449 shapes suggest that the gas circulation patterns might be different depending on the type of

450 sand used for the experiment.

454

455

456

457

458
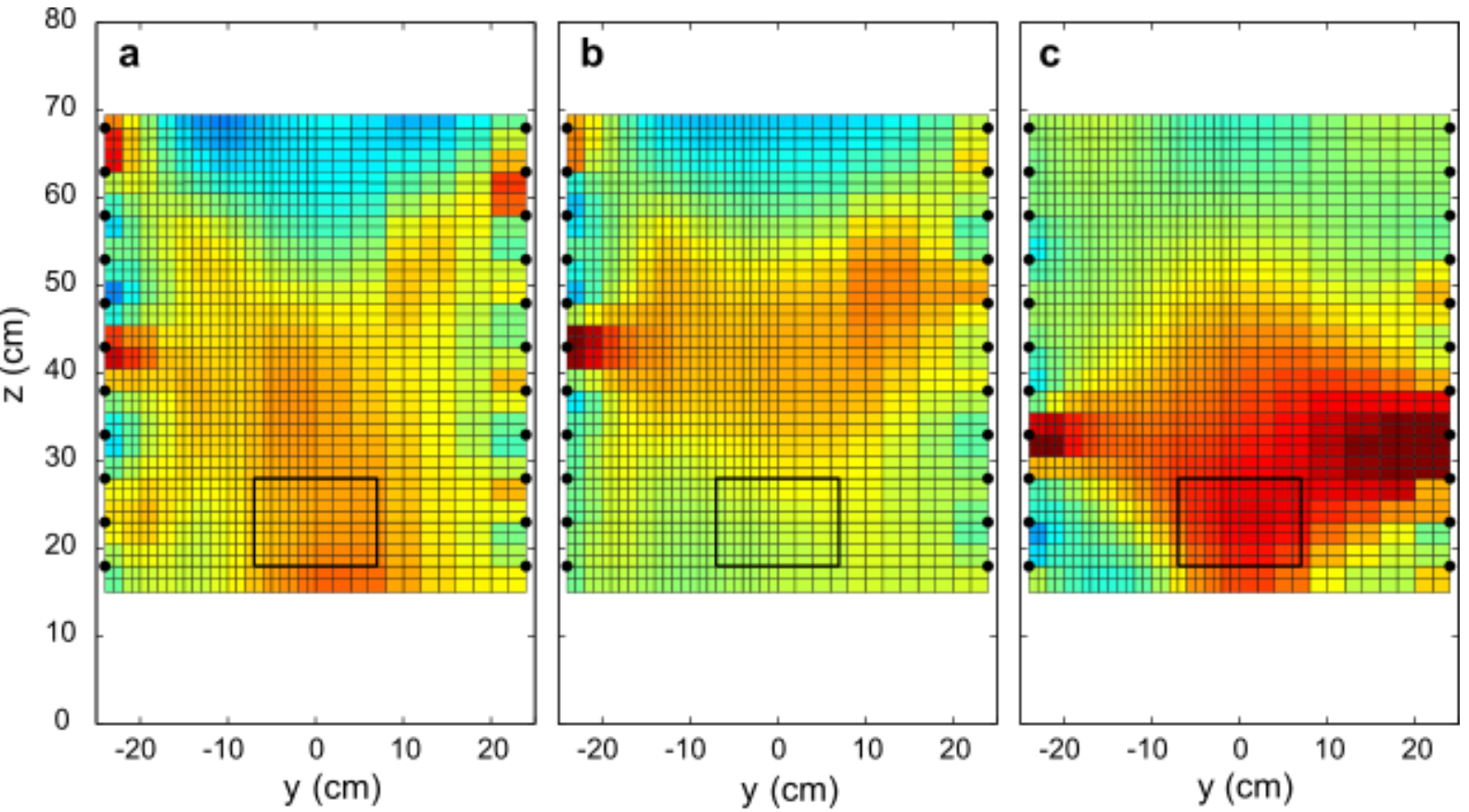

Resistivity (ohm.m)

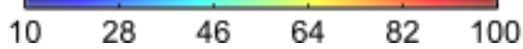

Fig. 7. Inverted resistivity distribution for gas injection in saturated silica sand. a: $\mathrm{N}_{2}$ injection, simple inversion. b: $\mathrm{N}_{2}$ injection, ratio inversion. c: $\mathrm{CO}_{2}$ injection, ratio inversion. The position of the air diffuser is given by the open rectangle. 


\subsection{Summary}

These results confirm that the ERT method is a suitable monitoring technique to detect gas injection into geological media. Yet, the shape and the magnitude of the resistivity anomalies seem to depend on the nature of the sand, the type of gas injected and probably on 471 the initial electrical conductivity of the saturating water. When the silica sand is involved, the anomaly seems to be restricted to the lower part of the tank, as if gas could not reach or accumulate in the upper volume. When the carbonate sand is used, the anomaly exhibits a ushape centered with the axis of the tank, and spreading from the top of the diffuser to the surface of the tank. Also, for a highly reactive system such as carbonate sand and gaseous $\mathrm{CO}_{2}\left(\mathrm{C} / \mathrm{CO}_{2}\right)$ the dominant physico-chemical processes are linked to the dissolution of the gas and of the solid matrix, leading to a decrease of the resistivity, as observed for example by Peter et al. (2012) or Vialle et al. (2014). Yet, for non-reactive or slightly reactive systems $\left(\mathrm{S} / \mathrm{N}_{2}, \mathrm{~S} / \mathrm{CO}_{2}, \mathrm{C} / \mathrm{N}_{2}\right)$, dissolution processes are weak and the dominant mechanism is the

480 desaturation of the porous space, which increases the resistivity of the medium, as observed 481 for example in the studies conducted by Nakatsuka et al. (2010), Breen et al. (2012) or Bosch 482 et al., (2016). In the following discussion, we want to address two main questions raised in view of 484 these experimental results. 1) In the case of slightly reactive systems, why does the increase in 485 resistivity exhibits different anomaly shapes depending on the type of sand involved. 2) Is it 486 possible to develop new measuring protocols that will further improve the ability of the 487 method to capture the shape and magnitude of these anomalies? 
Note that these two questions seem to overlook the case where dissolution processes dominate the electrical response of the system whereas it is relevant in many scenarios of $\mathrm{CO}_{2}$ leakage in the subsurface. We justify this choice because these particular cases have important temporal and kinetic aspects that are not addressed nor captured by the experiments presented previously, which rather focus on steady-state situations. However, they can both still bring pertinent answers to some of the possible $\mathrm{CO}_{2}$ leakage scenarios, as well as to any other 494 problems involving gas circulation in the subsurface or which demand the detection and delimitation of a resistivity anomaly.

In the next section, we will address question 1) and discuss how the sand grain size and the gas injection rate might be important factors controlling the geometry of the gas circulation plume, which results in varying resistivity anomaly shapes. We present a numerical model that was developed to challenge this hypothesis. It is structured as a stochastic cellular automaton and allows to simulating the injection of gas into saturated sands and computing the resulting electrical resistivity distribution of the medium. Finally, in section 5, we use this model to address question 2) and investigate the development of new measuring protocols that would improve the detection of these anomalies.

\section{MODELLING}

\subsection{The effect of grain size and gas injection rate on gas plume geometry.}

Apart from issues related to the geological storage of $\mathrm{CO}_{2}$, the way that gas spreads

508 into a saturated geological medium has also been studied during the development of the "air 509 sparging" method (Böhler et al., 1990; Johnson et al., 1993), a remediation technique which 510 consists of injecting air into or below a shallow contaminated area. While the air flows 511 towards the surface, the contact with the pollutant triggers biodegradation processes and 512 volatilization, allowing some of the pollutant to flow toward the surface under gaseous form, 
513 along with the injected air. The context of air-sparging is relevant here because it involves the

514 circulation of a nearly non-reactive gas (air), which is why many studies dealt with laboratory experiments very similar to those described in this study, especially those involving $\mathrm{N}_{2}$ 516 injection.

At the end of the 1990s, most of the authors working on the air-sparging method agreed that, in a saturated medium, the air circulates according to two main patterns. The "bubble flow" consists of a discontinuous circulation under the form of bubbles (Wehrle, 1990; Sellers and Schreiber, 1992); while the "channel flow" describes a circulation through continuous pathways, often called channels, which evolve independently from each other and are separated by zones fully saturated with water (Clayton, 1998; Elder and Benson, 1999).

523 The laboratory studies from Ji et al., (1993), Baker \& Benson (1996) and Brooks et al., (1999)

524 have shown that the main discriminating factor between these two flow modes is the grain 525 size of the porous medium. Brooks et al., (1999) gathered from several studies the different 526 kind of flow that can be observed depending on the mean grain size of the studied medium. 527 This comparison (see their figure 1) suggests that for a grain size below $1 \mathrm{~mm}$, the air 528 circulation follows the channel flow type, and for a grain size above $2 \mathrm{~mm}$ only the bubble 529 flow is observed. In between, a transition behavior is observed which consists of 530 discontinuous channels, and that later authors have been calling "mixed flow" or "slug flow". $531 \quad$ For thin sands $(\mathrm{d}<0.21 \mathrm{~mm})$, Peterson et al., (2001) identify a third type of gas flow 532 that they call "chamber flow". It involves the existence of continuous channels, which will 533 accumulate in some location of the porous medium, following mainly horizontal components, 534 hence forming gas accumulation "chambers" connected between each other through 535 continuous channels that are essentially vertically oriented. Brooks et al., (1999) have also studied the influence of the gas injection rate upon the 537 type of flow encountered. Their observations suggest that low injection rates favor the "slug 
538 flow" or "mixed flow" type, whereas high injection rates allow the gas to form continuous

539 channels. The different kind of flow observed depending on grain size and injection rate are

540 summarized in their table 1. Regarding the experiments described in this paper, the silica sand

541 and the carbonate sand maximum grain size are respectively $400 \mu \mathrm{m}$ and $2 \mathrm{~mm}$, and the

542 injection rate is high with respect to the references from Brooks et al., (1999). We can

543 therefore safely assume that the gas circulation during the experiments followed a "channel

544 flow" type or at the very least a "slug flow", and this assumption has been considered 545 throughout the following modeling work.

\subsection{Modelling gas injection into saturated sands}

The model developed in this paper has been built as a cellular automaton using

Matlab. A 3D parallelepiped matrix is generated, whose dimension is $d \times d \times h, d$ being the model consists of simulating gas circulation patterns through the cells, then defining an

553 effective water saturation value for each cell and finally estimating the associated resistivity

554 value using Archie's law (Archie 1942).

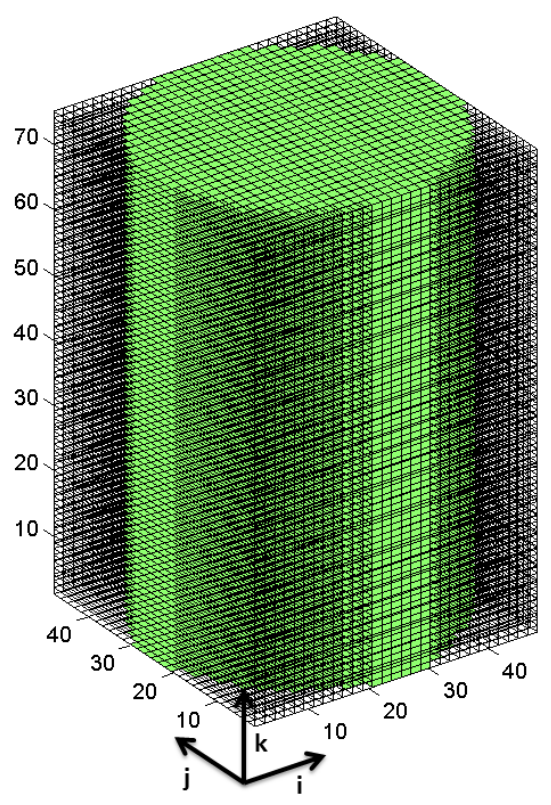

Fig. 8. Isometric view of the parallelepiped matrix. Green cells have been assigned the value 1 and represent the studied medium, while transparent cells have been assigned the value NaN and correspond to the outside of the tank. 


\subsubsection{Modelling gas circulation patterns}

As explained previously, based the grain size of the sand used during the experiments,

570 it can be considered that gas circulation follows the "channel flow" type. The first step of the

571 modeling process is hence to define the number of gas channels created during the injection,

572 their dimensions and their respective trajectory. Selker et al., (2007) describe the formation of 573 gas channels as a competition between restriction forces imposed by the capillary pressure 574 and buoyant forces produced by the density difference between gas and water. Assuming that 575 channels are cylindrical and that their extremity is hemispheric, Selker et al., (2007) define the 576 channels radius value to be

577

$$
R=\sqrt{\frac{3 \sigma}{\Delta \rho g}}
$$

where $\sigma$ is the interfacial tension between gas and water $(\mathrm{N} / \mathrm{m}), \Delta \rho$ is the density difference between gas and water, and $g$ is the gravity acceleration $\left(\mathrm{m} \cdot \mathrm{s}^{-2}\right)$. The computation of $R$ in the the channel diameter scale is similar to the scale of one cell of our cellular automaton grid. Selker et al., (2007) also propose an estimate of the number of gas channels created, that granular medium, respectively:

$$
N \approx \frac{Q \mu}{3 \pi \sigma \kappa}
$$


where $Q$ is the gas injection rate $\left(\mathrm{m}^{3} \cdot \mathrm{s}^{-1}\right), \mu$ is the dynamic viscosity of the gas $\left(\mathrm{kg} \cdot \mathrm{m}^{-1} \cdot \mathrm{s}^{-1}\right)$ and 589 591 about 100 channels for the carbonate sand.

592

593

594

595

596

597

598

599

600

601

602

603

604

605

606

607

608

609

610

611

612

$\kappa$ is the permeability to gas $\left(\mu \mathrm{m}^{2}\right)$ of the medium, that we estimate using the permeability to water values given in Table 1. Equation 9 yields about 300 channels for the silica sand and

\subsubsection{Stochastic evolution of channel trajectory}

Once the number and dimensions of gas channels have been estimated, the next step is to define the geometry of the gas plume, i.e the trajectory of the gas channels. Looking back at Fig. 5b, the resistivity anomaly suggests that the gas spreads following a parabolic shape or "u-shape", which has indeed been observed in several studies (Ji et al., 1993; Semer et al., 1998; Selker et al., 2007). Fig. 7, however, suggests that the gas is mainly concentrated in the lower part of the tank. This kind of behavior has been less frequently reported, but we assume in the following that it can be related to the "chamber flow" described by Peterson et al., (2001). Indeed, the chamber flow pattern has been reported for fine grain sands $(d<210 \mu \mathrm{m})$, which is approximately consistent with the grain size of the silica sand used in the experiment $(210 \mu \mathrm{m}<d<400 \mu \mathrm{m})$. It is therefore possible that the gas accumulation in the lower part of the tank consists of one "chamber" spreading laterally, and that gas is flowing toward the surface through vertical channels whose concentration is not high enough to produce significant resistivity anomalies. This hypothesis is supported by visual observations that were made during similar gas injection experiments using the same silica sand (see Kremer et al., 2016a and their Figure. 9b).

Our first objective in this modeling work is to implement the parabolic shape gas circulation mode and the "chamber" flow mode, to see if they can respectively explain the resistivity anomalies observed during experiments $\mathrm{C} / \mathrm{N}_{2}$ and $\mathrm{S} / \mathrm{N}_{2}$. For Selker et al., (2007), 
613 the parabolic circulation pattern can be explained by the fact that in a relatively isotropic

614 medium, the probability that a gas channel moves toward one lateral direction or another is

615 the same. Therefore, the probability distribution associated with the position of a gas channel

616 in the horizontal plane follows a Gaussian law, and the channel concentration is maximal at

617 the injection point.

618 To model this behavior, a stochastic approach has been adopted. It consists of 619 simulating the trajectory of each gas channel independently as the successive invasion of 620 neighboring cells. Each channel begins at a cell chosen randomly just above the gas diffuser. 621 The trajectory of the channel is then constrained to 2 possibilities: 1) move up in one of the 622 cells located above which share at least one edge with the present cell (9 possibilities); 2) 623 move laterally in one of the cells sharing at least on edge with the present cell (8 possibilities).

624 The first probabilistic event "gas moves vertically" is named $\mathrm{V}$, of probability $P_{V}$, while the 625 second probabilistic event "gas moves horizontally" is named $\mathrm{H}$, of probability $P_{H}$. As the universe is restricted to these two events we have

$$
P_{V}=1-P_{H}
$$

628 For a given value of $P_{H}$ (or $P_{V}$ ), a selection with equal probability is performed to determine 629 whether the channel moves vertically or horizontally. Then another selection is done to 630 determine which of the 8 (or 9) cells will be invaded. This is repeated until a cell at the top of 631 the tank is reached. After that, we consider that the channel is stable and that gas is flowing 632 out of the tank. This stochastic process is applied independently to each gas channel created. 633 At the end of the simulation, the number of channels passing through each cell is recorded. 634 The shape of the gas plume is then controlled by the value of $P_{H}$, as shown on Fig. 9 where, as expected, high $P_{H}$ values result in a wider gas plume, with a higher number of channels in the 636 middle part. 


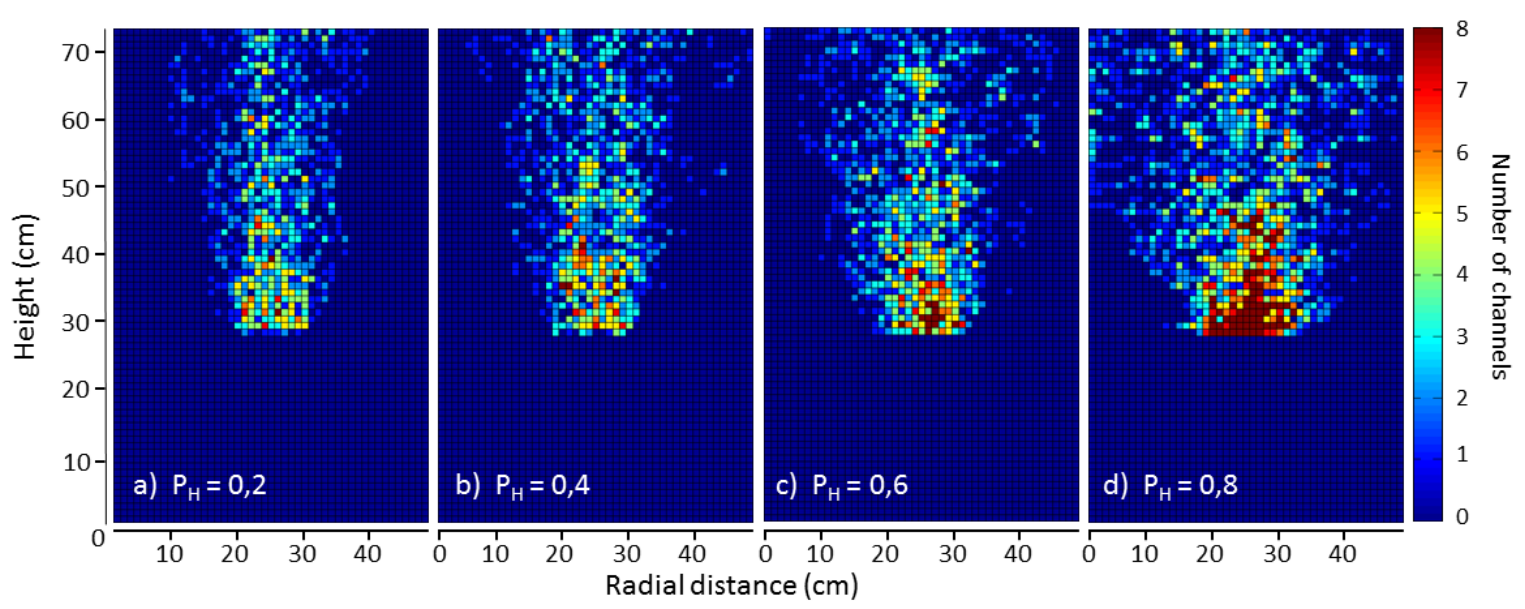

642 Fig. 9. Vertical cross-sections of the parabolic shape circulation model, for several values of probability $\mathbf{P}_{\mathrm{H}}$. The color code represents the total number of channels passing through each cell.

644 This way of simulating gas channel evolution preserves the Gaussian distribution of pathways

645 described by Selker et al., (2007), as shown in Fig. 10a. To model the chamber flow

646 accumulation describe by Peterson et al., (2001), the same stochastic approach is used, except

647 that the probability $P_{H}$ is no longer fixed, but is a function of the vertical position of the 648 current cell.

$$
P_{H}=\left(1-\frac{z-z_{\text {diff }}}{h-z_{\text {diff }}}\right) \cdot e^{\frac{z-z_{\text {diff }}}{z_{\max -z_{\text {diff }}}}}
$$

650 where $z$ is the actual position of the channel extremity, $z_{\text {diff }}$ is the diffuser vertical location

651 and $h$ denotes the height of the surface. Such a definition implies that the probability of

652 horizontal movement is high close to the diffuser location and decreases exponentially as the

653 channel evolves toward the surface. It results in channel accumulation in the lower part of the

654 tank, while the channel density is lower in the upper part (see Fig. 10b and 10c), thus

655 reproducing the concept of "chamber flow accumulation". 

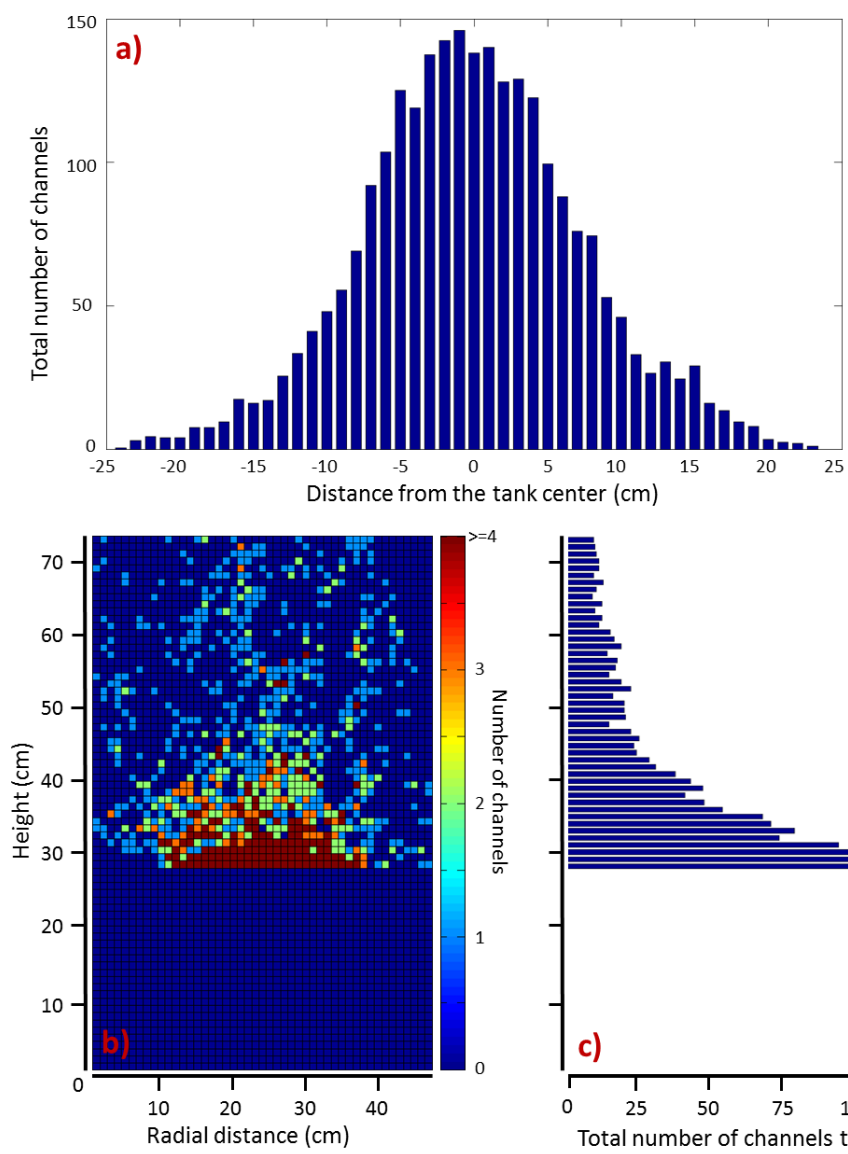

Fig. 10. a) Histogram representing the lateral distribution of channels in the parabolic shape model. The distribution is globally Gaussian. b) Vertical cross-section of the accumulation chamber model, and c) corresponding vertical distribution of channels.

\subsubsection{Defining saturation level and resistivity values}

676 As explained previously, given the definition of gas channel dimensions (equation 6), the

677 channel diameter is approximately of the same order as the model cell size. Therefore, we

678 consider in the following that several channels passing through the same cell only result in

679 one channel passing through the cell. A fixed gas saturation value $S g$ is then attributed to all

680 cells invaded by gas. This value is chosen randomly between 0.4 and 0.5 , as observed by

681 Chen et al., (1996). The saturation values distribution is then smoothed by averaging the value 
683 calculated using Archie's law (Archie, 1942). The porosity values of the sands are known, and

684 the cementation exponent $m$ was chosen to be 1.3 (unconsolidated sands). Note that surface

685 conduction is not taken in account with Archie's law, which is acceptable given the

686 conductivity value of the fluid which is in the range where surface conduction is negligible

687 compared to bulk conduction (Glover, 2010). Fig. 11 displays the vertical sections of the

688 saturation values obtained with the model and the associated resistivity distribution for the

689 "parabolic" circulation case and for the "chamber accumulation" case.
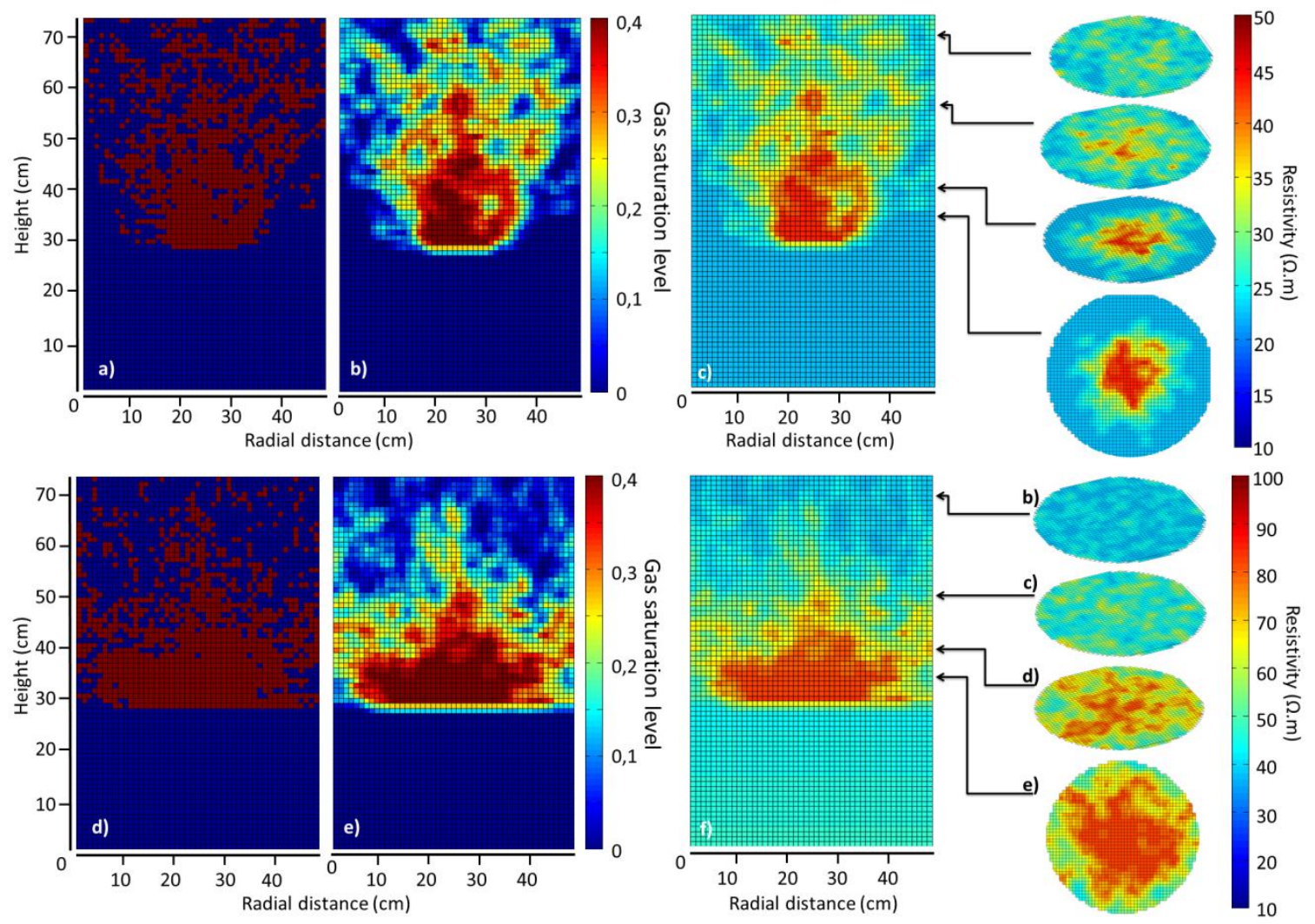

Fig. 11. Vertical and horizontal cross-sections representing the distribution of channels, saturation values and resistivity values, respectively, for the parabolic circulation model (a,b,c) and the chamber accumulation model (d,e,f). 
698

699

described in appendix A. They are key parameters that allow modelling different gas plume geometries and reproduce those observed during the experiments. In order to model the injection of $\mathrm{N}_{2}$ into the carbonate sand, we use probabilistic parameters that produce a u-shape (or parabolic) gas plume (Fig. 11a), as suggested by the experimental results (Fig. 5 and 6), and as observed in several studies about gas injection into sands with similar characteristics (Ji et al., 1993; Semer et al., 1998; Selker et al., 2007). In the case of $\mathrm{N}_{2}$ injection into silica sands, the resistivity shape observed in the experiments reminds the "chamber flow" geometry observed by Peterson et al. (2001) on similar fine grain sands. The probabilistic parameters are therefore adapted to reproduce this specific geometry (Fig. 11d). Then, gas saturation values are assigned based on Chen et al., (1996), smoothed by averaging with the neighboring cells (Fig, 11b and 11e), and resistivity values are finally computed using Archie's law (more detail in appendix A). The results are 3D representations of the resistivity distribution within the tank, as shown on Fig. 11c and 11f.

\subsubsection{Comparing model and experimental results.}

The software R3t was then used to: 1) calculate the forward model associated with these two synthetic resistivity distributions, considering the same electrodes locations and measuring protocol (cross-rods) as during the experiments; 2) calculate the inverse model using the so computed synthetic resistances. Fig. 12 compares the inversion results obtained based on the synthetic models ( $b$ and d) with those obtained based on experimental measurements (a and c). The resistivity range is very similar in both cases (between 60 and $90 \Omega \cdot \mathrm{m}$ ), and the shapes of the high resistivity anomalies also match, thus confirming that in silica sands, the gas is likely to be circulating following the chamber accumulation flow type whereas in carbonate sands, the parabolic gas plume is a plausible description of the gas circulation patterns. 

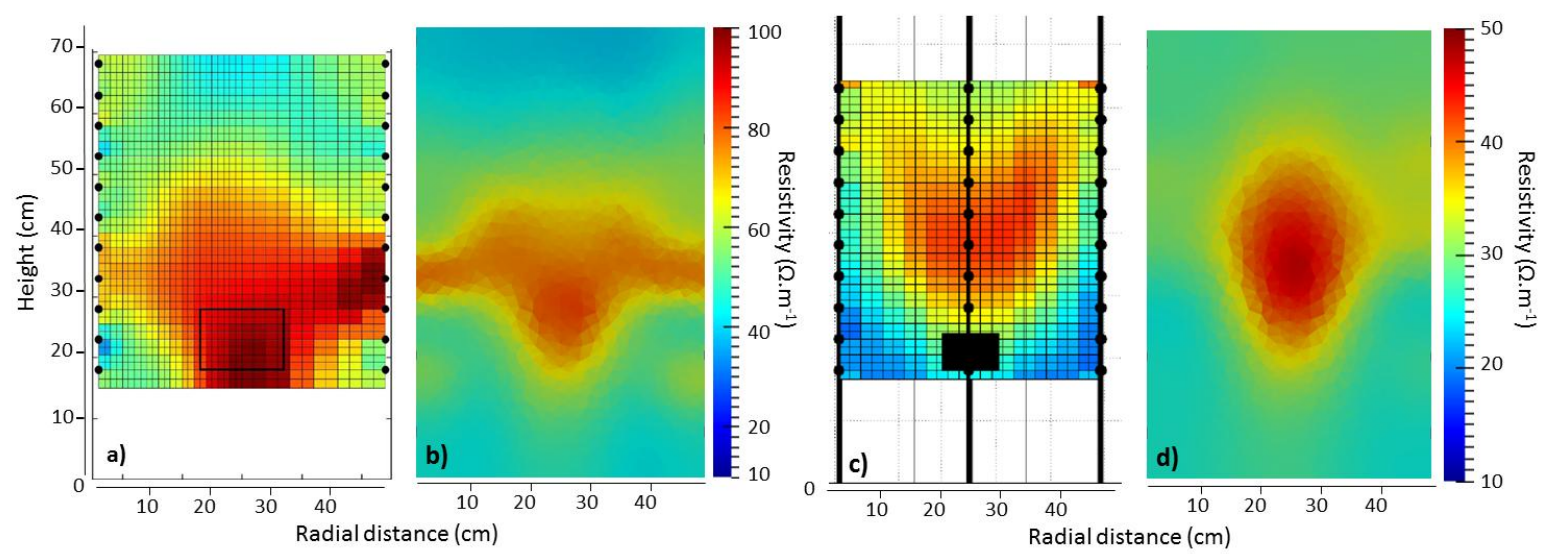

723

Fig. 12. Comparison between the inversion results obtained from the experimental data and from the cellular model, for the accumulation chamber $(a, b)$ and the parabolic cases $(c, d)$, respectively.

These results suggest that, in saturated sands, the grain size distribution is likely to be the main factor controlling the shape of the gas circulation patterns, and hence of the resistivity anomaly that is observed. The differences between the observed shapes are quite significant. Grain size distributions that result in high permeability values (e.g. $16.5 \mu \mathrm{m}^{2}$ for carbonate sand) will favor the occurrence of u-shaped gas plumes that have a strong vertical component. For lower permeability values (e.g. $4,5 \mu \mathrm{m}^{2}$ for silica sand), the gas plume takes the form of an accumulation chamber with dominating horizontal components. One could therefore expect that grain size variation within an aquifer will produce specific resistivity anomaly shapes alternatively dominated by vertical and horizontal orientations. These observations highlight the fact that knowing the grain size distribution of sandy aquifers is very important if one wants to anticipate the shape of the gas circulation patterns, and hence of the prospective resistivity anomaly that would be observed through ERT monitoring of the aquifer.

\section{DISCUSSION}

In light of these results, we investigated the possibility to develop new measuring protocols that would improve the capacity of the ERT method to detect and capture the shape 
743 and magnitude of the resistivity anomalies produced by gas circulation into saturated sands.

744 Indeed, even if some information is available to help anticipate the shape of the expected

745 resistivity anomaly, it is possible that different cross-hole protocols would not be equally

746 efficient into detecting and retrieving the gas plume geometry because of their different

747 sensitivity characteristics.

748 As a reminder, the cross-rods protocol was initially chosen to perform the experiments

749 because it was proven to be more efficient than the bipole-bipole, dipole-dipole and

750 Schlumberger protocols (see section 2). Here we attempt to design even more efficient

751 protocols than the cross-rods. To do so, our approach consists of analyzing the sensitivity

752 distribution of all the electrode configurations that composed the cross-rods protocol, so as to

753 combine them in a new, more optimal organization. The respective efficiency of these new

754 protocols is then tested using the cellular automaton model, by simulating rough synthetic

755 resistivity distributions as well as realistic gas circulation patterns.

756

\subsection{Electrode configurations families.}

The cross-rods protocol consists of performing all possible electrode combinations for 759 current injection and voltage measurement, in each of the 10 planes (rings) of electrodes 760 instrumenting the tank. Electrodes from different planes are never used together for a single

761 measurement. This protocol hence results in several electrode geometric combinations. To be 762 more specific, as a plane contains 6 electrodes, the number of combinations possible for 4 763 electrodes measurements yields 45 possibilities (not including reciprocals). Among these 45 764 possibilities, one can distinguish 8 different geometric families, which are represented in Fig. 76513 and that we name "classes" 1 to 8. 

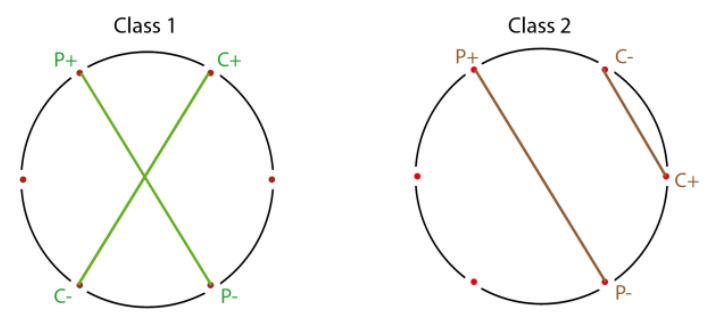

772

773
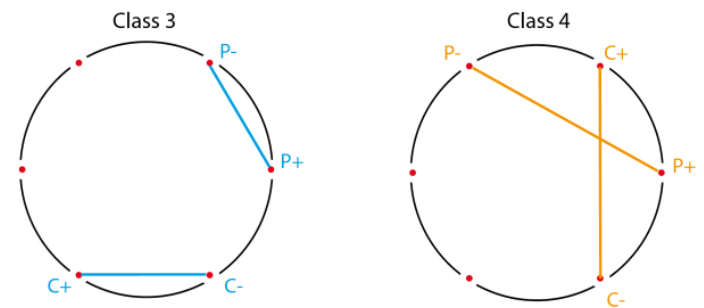

776
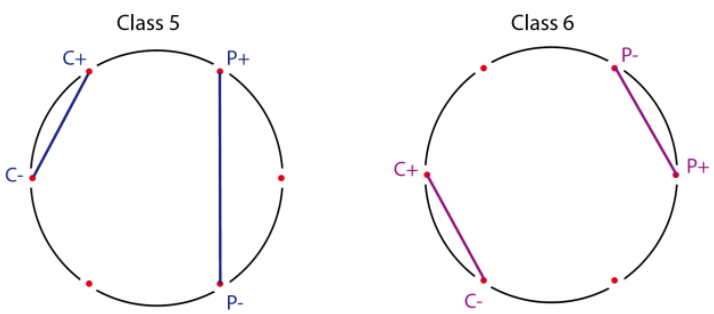

779
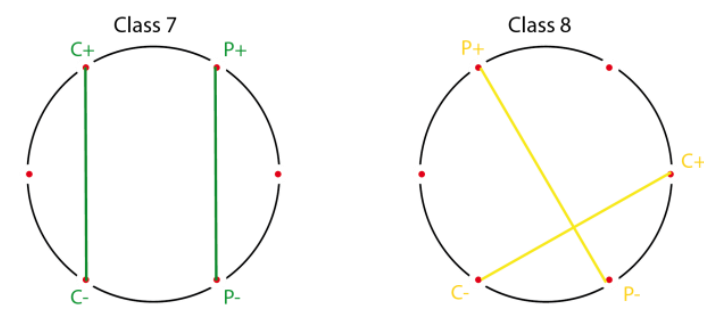

Fig. 13. Schematic representation of the different four electrodes arrays families encountered in the cross-rods protocol.

\subsection{Sensitivity distribution of the different families.}

787 Given the different geometries of these electrodes combinations, they present different 788 sensitivity distributions that we chose to estimate using the following procedure. The

789 objective is to perform forward modeling using the cross-rods protocol on two cylindrical 790 media (representing the cylindrical tank) to which a different resistivity distribution is applied.

791 We attributed to the first medium a homogeneous resistivity distribution of $25 \Omega \cdot \mathrm{m}$ (same 792 order of magnitude than the saturated sand). The second medium has the same background 
793 resistivity value in which is included a more resistive cylinder $(100 \Omega \cdot \mathrm{m})$ whose axis is

794 identical or parallel to the cylindrical tank axis. For each electrode configuration class, the 795 resistances obtained through forward modeling are then compared by computing the relative 796 resistance variation percentage between the "cylindrical intrusion" model and the 797 homogeneous model:

798

$$
\partial R_{\%}=\frac{\left|R_{c y l}\right|-\left|R_{\text {hom }}\right|}{\left|R_{\text {hom }}\right|}
$$

799

800

801

802

803

804

805

806

807

808

809 configurations used in the cross-rods protocol.

where $R_{c y l}$ and $R_{\text {hom }}$ are the resistances values obtained through forward modeling on the cylindrical intrusion model and the homogeneous model, respectively. Generally speaking, the sensitivity distribution quantifies how much a given resistance measurement is affected by a change of resistivity in the medium. Here, the parameter $\partial R_{\%}$ can be seen as a "macroscopic" estimation of the sensitivity since it quantifies how much the resistance measured is affected by the presence of a resistive cylinder. Then, by displacing the cylindrical intrusion into the (xy) plane, and repeating this forward modeling comparison, one can quickly obtain a 2D mapping estimation of the sensitivity distribution in the (xy) plane. These sensitivity distributions were estimated for each electrode geometric configurations and regrouped in Fig. 14, where high relative variations values denote a higher sensitivity area. The resolution of this mapping is relatively poor (resolution equivalent to the cylindrical intrusion diameter), but was very quick to implement, and nevertheless yields quantifiable and valuable information regarding the sensitivity behavior of the different electrodes 

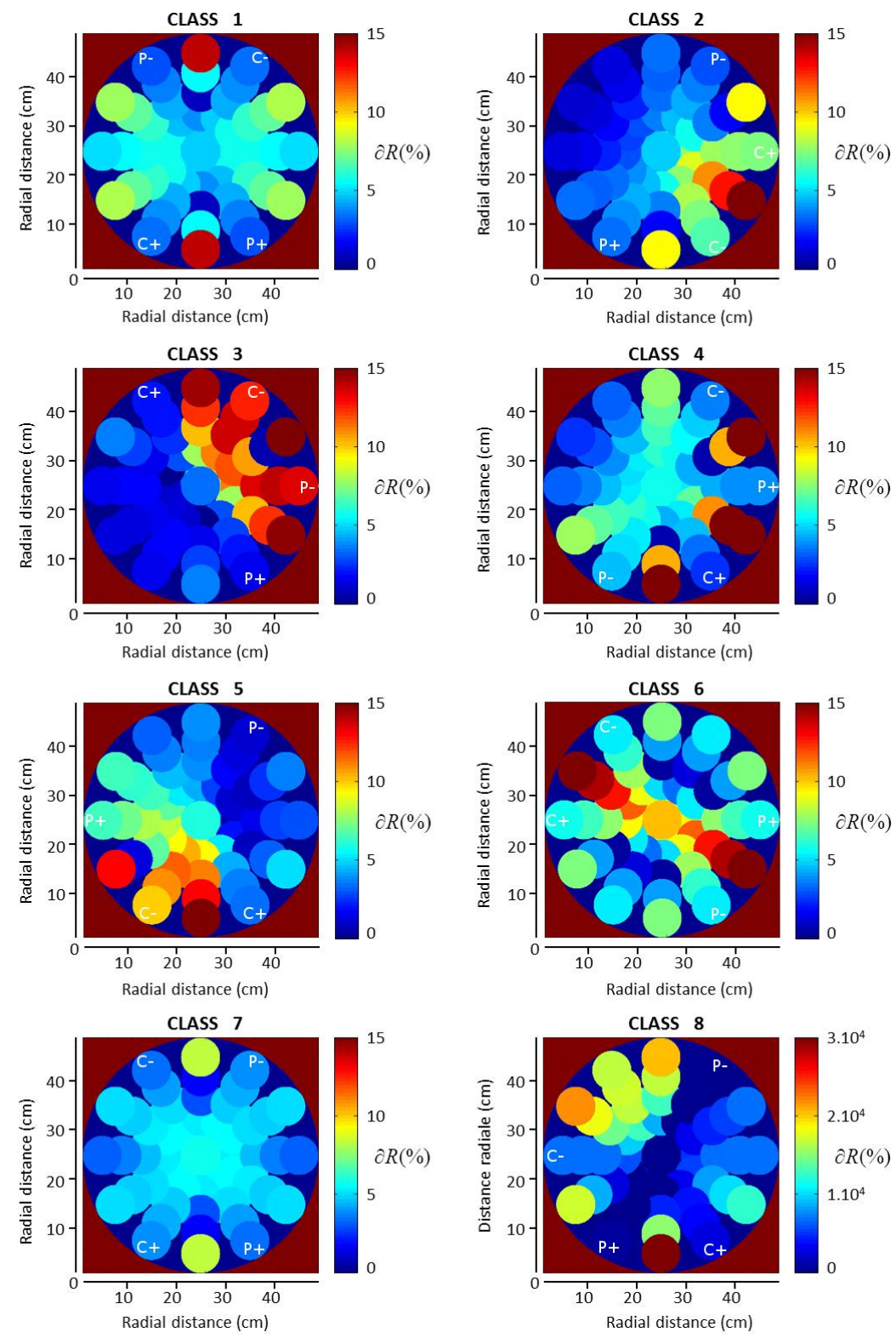

Fig. 14. Sensitivity distributions obtained for each class of electrode array geometry. Letters $\mathrm{C}-, \mathrm{C}+$ and $\mathbf{P}+-, \mathrm{P}+$, denote the current injection electrodes and potential measurement electrodes locations, respectively.

840 Fig. 14 shows that the geometries can be separated into two different groups. Geometries $n^{\circ} 1$, 
symmetrical with respect to the center of the tank (excepting ${ }^{\circ} 4$ ). Among them, geometry

$843 \mathrm{n}^{\circ} 6$ is of particular interest since it presents the highest sensitivity values at the center of the

844 tank, and high values (> 10\%) on a diametrically symmetrical fringe. Another group

845 composed of geometries $n^{\circ} 2,3,5$ and 8 , rather show high sensitivity values at the lateral areas

846 of the tank. In particular, geometry $n^{\circ} 3$ has the highest values distributed over approximately

847 a third of the tank circular surface. This suggests that, taken together, the 3 electrode arrays

848 that possess geometry $\mathrm{n}^{\circ} 3$ provide a high sensitivity scan of the entire tank surface, except in

849 the center.

Another electrode configuration which deserves special attention is geometry

$\mathrm{n}^{\circ} 8$. This specific set-up yields extremely high relative variations compared to the others (in

852 the order of $10^{\wedge} \%$ ). This is due to the numerical nature of the study, and is directly related to

853 the fact that the injection and measuring dipoles are perpendicular. Because of this, in a

854 perfectly homogeneous medium, perpendicular dipoles imply that the current lines and the

855 equipotential lines are perpendicular, yielding a null potential difference and therefore a null

856 resistance. In our case, the obtained resistance is not exactly 0 because of the irregularities of

857 the mesh (the tank is not perfectly cylindrical), but remains very small (of the order of $10^{-3}$

$858 \mathrm{Ohm})$. Then, including a different resistivity object in the environment still leads to the

859 measurement of small resistances, in the order of $1 \mathrm{Ohm}$, which results in a relative variation

860 of several order of magnitude. Even though these considerations depend on the numerical

861 nature of this study, it has also been observed in the real case that the relative variations

862 induced by gas injection in a relatively homogeneous medium are higher for the

863 measurements associated with this perpendicular electrode geometry. In the following

864 sections, we investigated further this specific configuration (and others), to verify if this

865 specific behavior could play an important role in the reconstruction of resistivity contrasts and

866 anomalies. 


\subsection{Designing new protocols}

In this section we present several numerical tests performed in order to explore the

870 behavior of some specific electrodes geometries and assess their influence on the quality of

871 the inversion results. We also aim at creating new protocols that may possess an improved

872 sensitivity and resolution compared to the cross-rods protocol. To do so, we performed a

873 series of tests, which consisted of applying different protocols for the computation of the

874 forward and then inverse problem, on specific resistivity distributions composed of a high

875 resistivity object $(100 \Omega \cdot \mathrm{m})$ included into a lower resistivity background $(25 \Omega \cdot \mathrm{m})$ and

876 comparing the quality of the inversion problem results between each protocol. The high

877 resistivity object was chosen as a cross, composed of two perpendicular parallelepiped

878 volumes (Fig. 15), so as to assess the capacity of the tested protocol to retrieve both horizontal

879 and vertical structures.

880

881

882

883

884

885

886

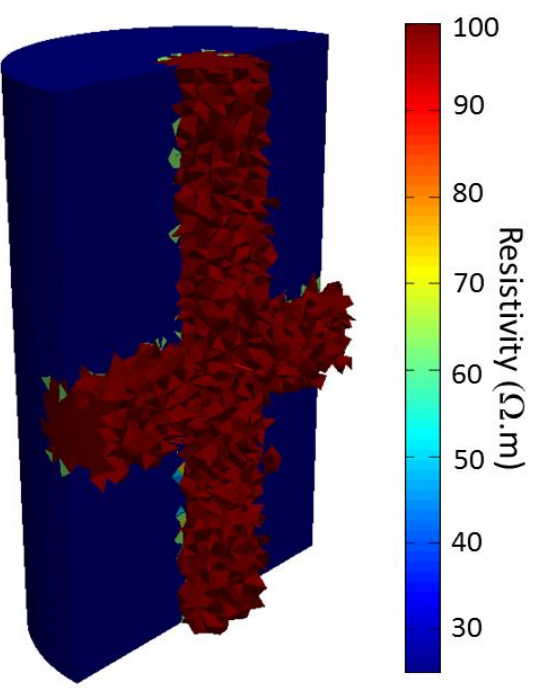

888

Fig. 15. Representation of the cross-shape resistivity intrusion embedded into the homogeneous resistivity cylindrical medium. 
In order to investigate the importance of each of the 8 electrode geometries on the global

892 performance of the cross-rods protocol, we performed a first test consisting of removing all

893 measurements associated to a specific electrode geometry and compare the ability of these

894 "truncated" protocols to retrieve the cross shaped object. The results are presented on Fig. 16.

895 As expected, removing some measurements from the protocol deteriorates the quality of the

896 inversion results. However, the degree of deterioration varies depending on which electrode

897 geometry is excluded. Geometries $\mathrm{n}^{\circ} 1,2$ and 3 seem to play an important role since their

898 removal results in a strong weakening of the results quality compared to the full cross-rods

899 protocol. The worst result seems to be obtained when geometry $n^{\circ} 3$ is absent from the 900 protocol. Geometry $\mathrm{n}^{\circ} 6$ may be the most important one, since without the associated

901 measurement the inversion process cannot converge.

902

903

904

905

906

907

908
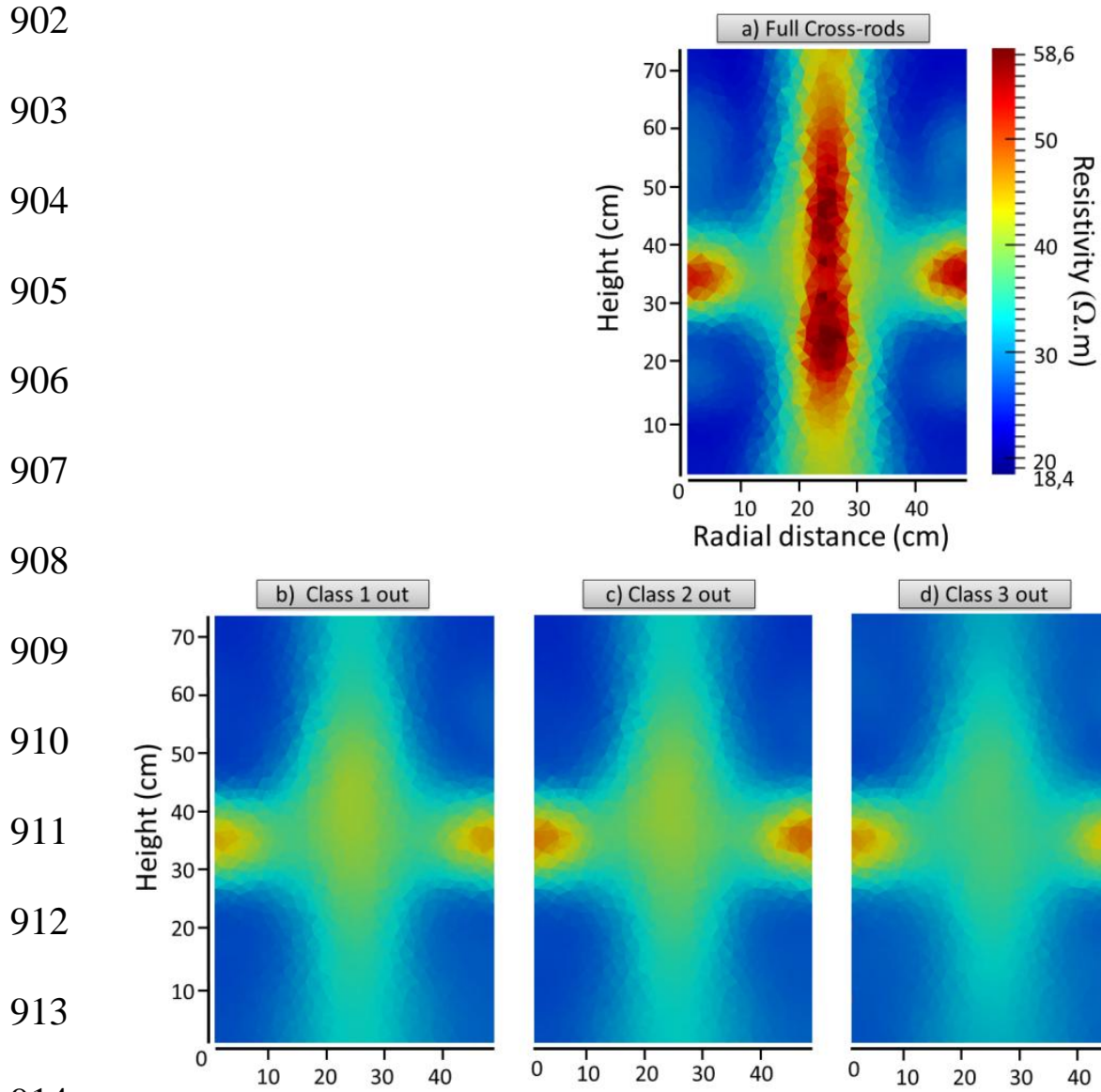

914
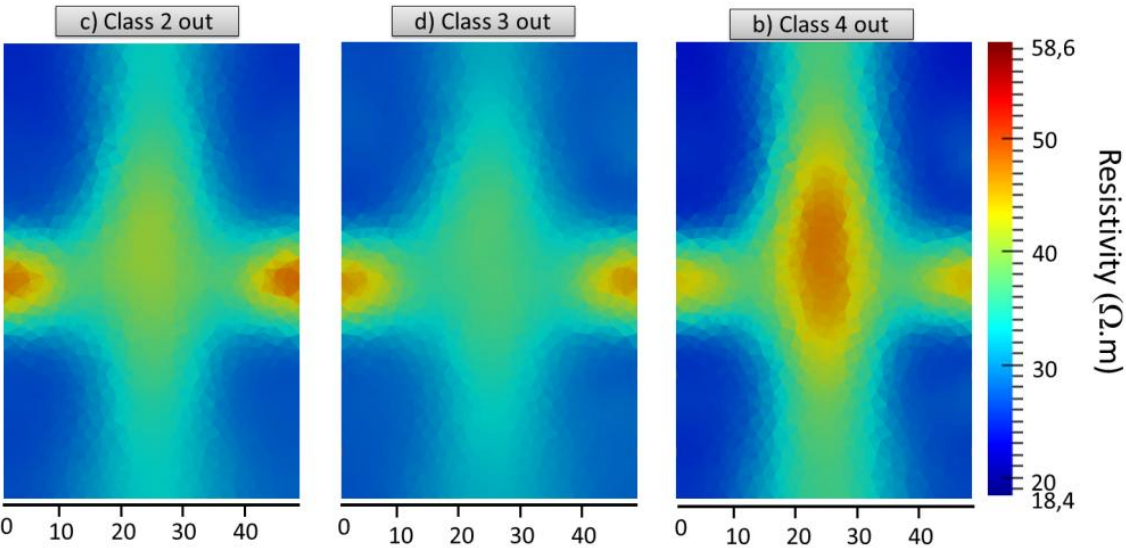

915
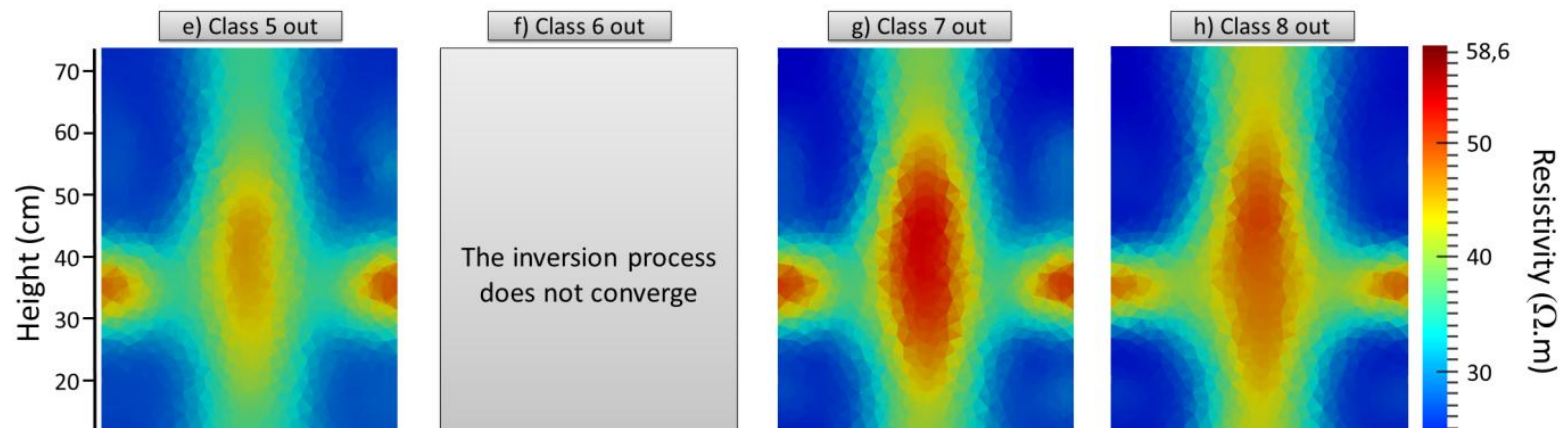
Test 2: measurements with electrodes in different planes.

922 The purpose of this test is to analyze the contribution of measurements involving electrodes 923 from different planes. Given the results from the previous test, we chose to develop these 924 inter-planes protocols using electrode configuration geometries $n^{\circ} 3$ and $n^{\circ} 6$. We define an 925 inter-plane measurement as an electrode configuration where one current injection electrode 926 and one potential measurement electrode are located in a different plane from the two other 927 electrodes. Fig. 17a illustrates this definition. It shows that one electrode geometry can be 928 derived in 9 additional measurements (without considering reciprocals). A protocol was designed which included all the inter-planes measurements possible using geometries $\mathrm{n}^{\circ} 3$ and $\mathrm{n}^{\circ} 6$. However this protocol gathered 900 measurements, when the initial cross-rods protocol

931 has only 450 measurements. In order to have a comparable number of measurements and thus 932 truly compare the global efficiency of the protocol, we designed a split inter-planes protocol, 933 for which each initial geometry is not derived in 9 additional measurements but in 4, 934 depending on the plane position of the initial measurement (Fig. 17b). By doing so the split 935 inter-planes protocol is composed of 576 measurements, which is closer to the number of 936 protocols composing the cross-rods protocol.

a) Full inter-plan protocol

938

939

940

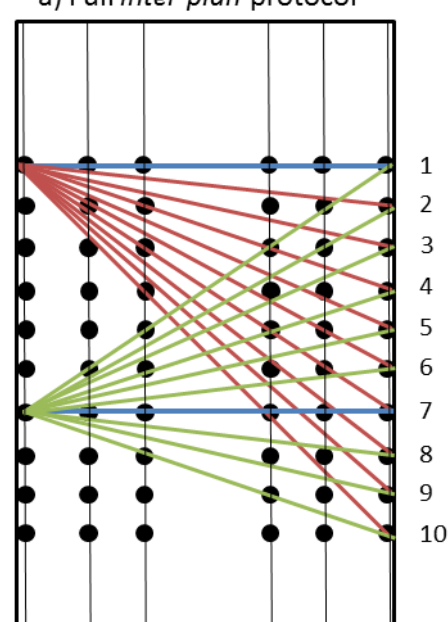

b) Split inter-plan protocol

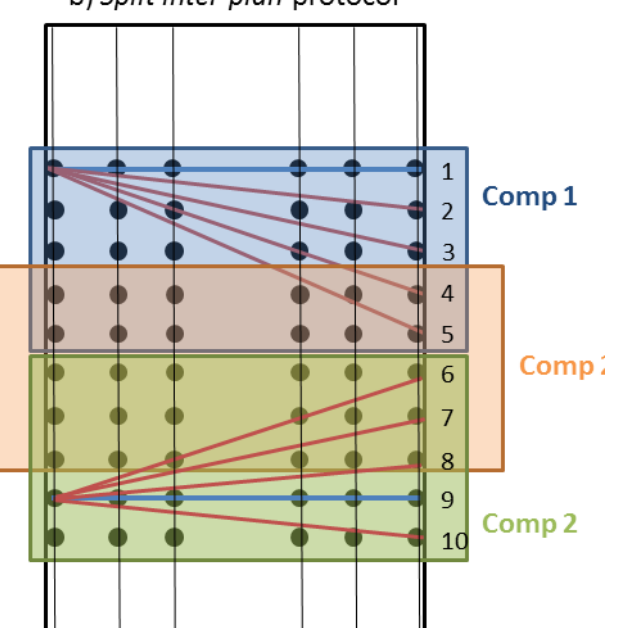


Fig. 18 compares the results of the "cross shape" test for the cross-rods, the inter-

948 planes, and the split inter-planes protocols. We clearly observe that the inter-plane protocol

949 gives the best results. The resistivity values are higher where the cross shape is supposed to be 950 reconstructed, and the horizontal part of the shape is better retrieved with inter-planes 951 protocols than with the cross-rods one. For a smaller amount of measurements (split inter952 plane protocol), the results are slightly worse but still remain much better than the cross-rods 953 protocol.

954

a) Cross-rods protocol

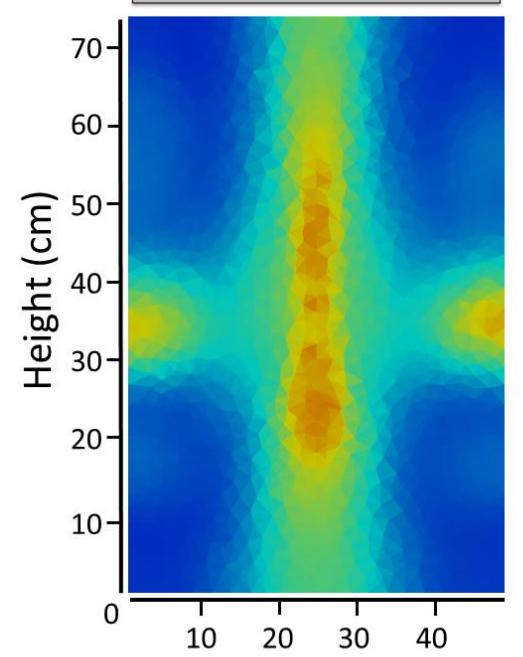

b) Inter-planes protocol

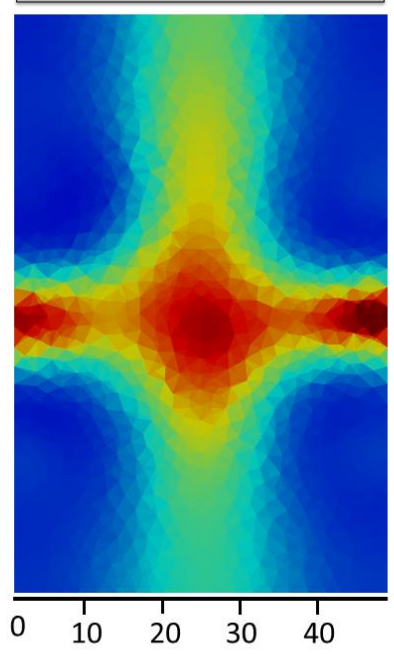

Radial distance $(\mathrm{cm})$

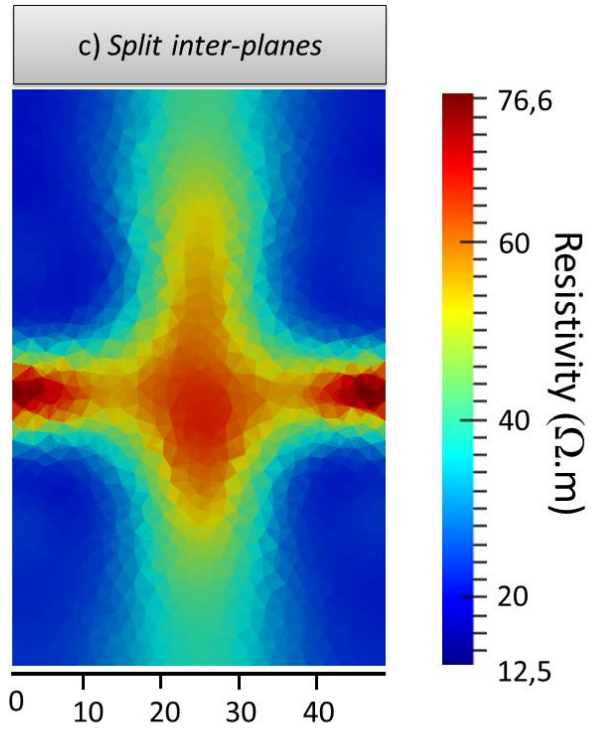

$\begin{array}{lllll}0 & 10 & 20 & 30 & 40\end{array}$

Fig. 18. Results from test 2. Comparison between the cross-shape test using a) the cross-rods protocol (450 measurements), b) the inter-planes protocol (900 measurements) and c) the split inter-planes protocol (576 
967 Based on these results, we worked on building protocols using measurements from the cross968 rods protocol and from the inter-planes protocols associated with geometry 6 and 8 . As it is 969 quite difficult to identify which specific configuration brings the most information, we chose 970 as a first step to build protocols with 450 measurements by randomly selecting 150 electrodes 971 configurations in the cross-rods and in the 6 and 8 inter-planes protocols. We generated 10 972 different protocols and compared their results to the cross-shape test. Only 3 over 10 produced better results than the cross-rods protocol, among which one showed particularly good results that we named Random. By studying which electrode geometries were picked-up initially, we noticed that these 3 protocols have in common a high amount of measurements from geometry 2, 3, 4, 5, 6 and 8, and a low amount from geometry 1 and 7. Considering that the random pick-up from 6 and 8 inter-planes protocol already bring a sufficient amount of geometries 6 and 8, we chose to restrict our random pick up in the cross-rods protocol to geometries 2, 3, 4 and 5, in proportions of 30,60, 30 and 30 measurements, respectively. Among the 10 protocols generated with this method, 8 demonstrated a superior behavior at 981 the cross-shape test than the cross-rods protocol, and one called the Random Constrained was 982 selected as the most efficient.

\section{Test on new geometries and realistic resistivity distribution}

From the three tests previously described, three protocols emerged which showed a better

986 capacity than the cross-rods protocol to reconstruct the cross-shape object: the split inter987 planes, the Random, and the Random constrained protocols. Fig. 19 compares the ability of 988 these protocols to reconstruct other geometries such as a non-centered cross (Fig. 19a) and a 989 diagonal rod (Fig. 19b). In these two cases, the three new protocols still produce a better 990 restitution of the initial resistivity distribution, and the highest resistivity values are reached 
991 with protocol Random constrained. Fig. 19c and 19d compare the results of forward modeling

992 and inversion processes performed on resistivity distributions that represent the "chamber

993 flow" and "u-shape" gas plumes simulated with the cellular automaton model. In these cases,

994 results are not anymore so consistent. The Random constrained protocol still demonstrates a

995 satisfying behavior ( $2^{\text {nd }}$ best for both distributions), but is not anymore the most efficient. For

996 the chamber flow distribution, the Random protocol is superior, whereas for the u-shape

997 distribution the cross-rods protocol is the most efficient protocol. These results demonstrate

998 that the efficiency of the protocol to detect a gaseous plume greatly depend on the geometry

999 of the plume. Here we found that Random constrained seems to be quite polyvalent whereas

1000 Random and cross-rods are respectively the best choices to image a "chamber flow" or a " $\mathrm{u}$ -

1001 shape" geometry.

1002
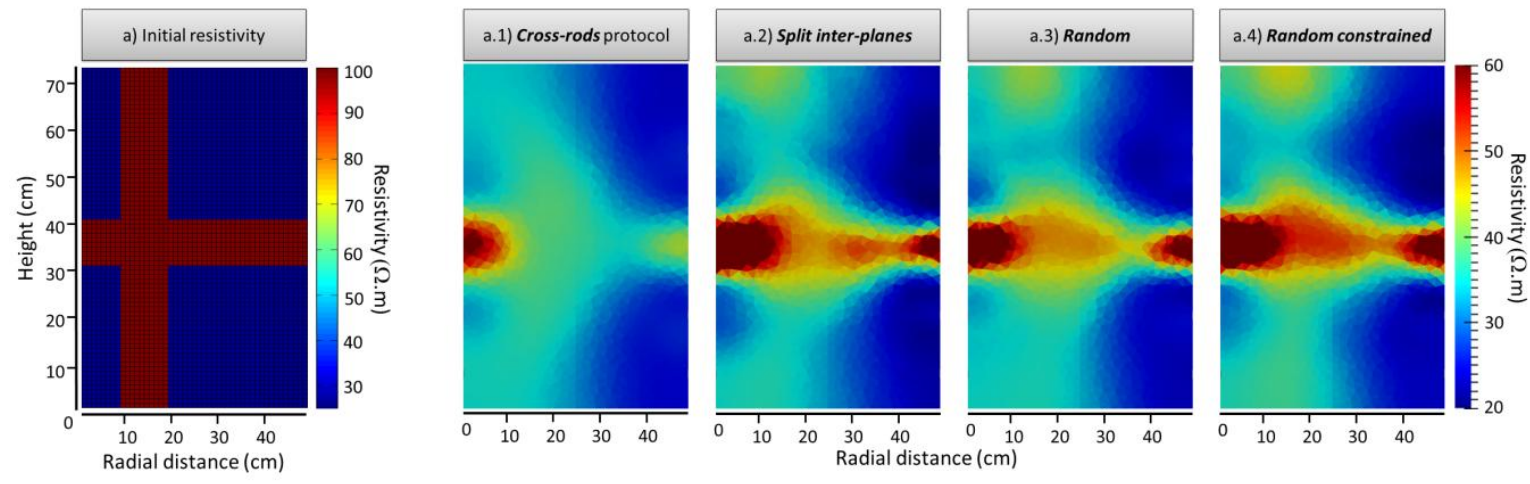

1007

1008

1009

1010

1011

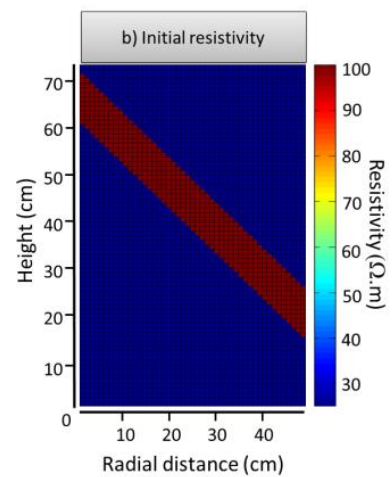

b.1) Cross-rodsprotocot
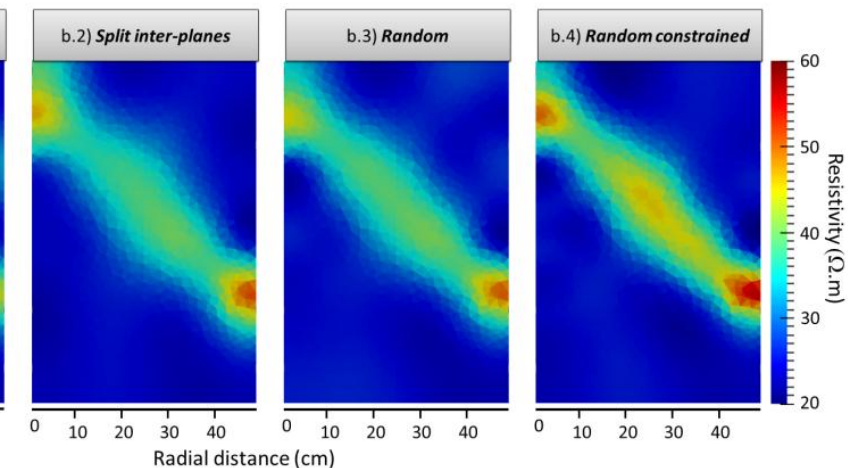

1012

Radial distance $(\mathrm{cm})$
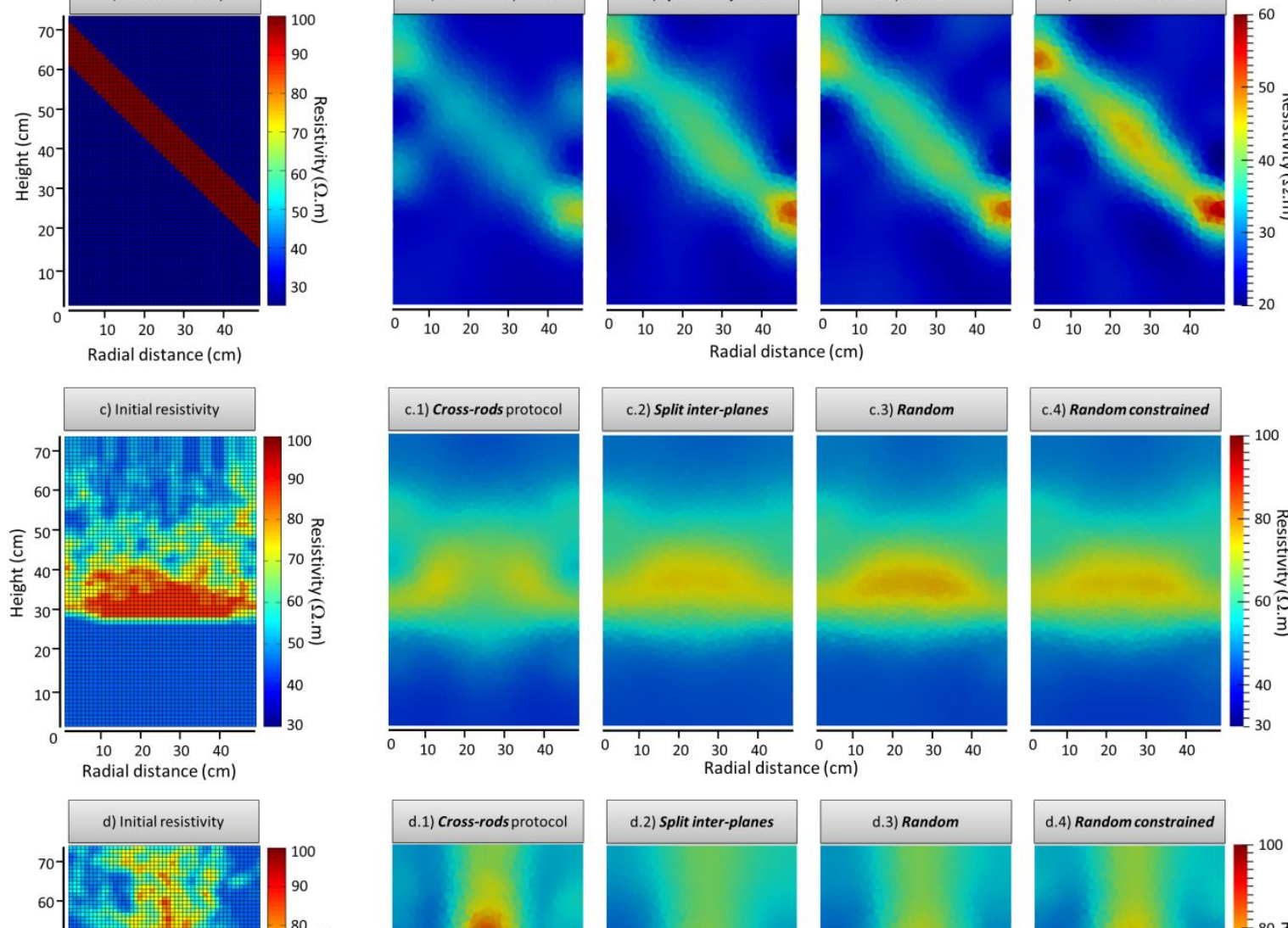

d.1) Cross-rods protocol
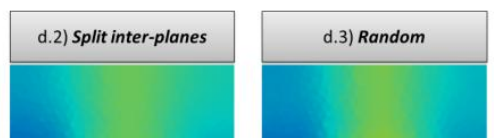

d.4) Random constrained

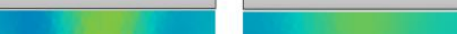




\section{CONCLUSION}

The laboratory experiments confirmed the ability of the ERT method to detect, locate

1026 and image $\mathrm{CO}_{2}$ and $\mathrm{N}_{2}$ plumes into water saturated sands. The experiments validated the 1027 occurrence of two different effects leading to opposite resistivity variations compared to the background distribution: the "insulating phase" effect and the "dissolution processes" effect. Also, the resistivity anomalies produced by $\mathrm{N}_{2}$ injection exhibit different shapes depending on the type of sand used during the experiments. Parabolic shapes are observed with carbonate

1031 sands, whereas more flattened shapes are observed with silica sand.

1032 Using a gas circulation model designed as a cellular automaton with stochastic 1033 behavior, we managed to reproduce the resistivity distributions observed during the 1034 experiments involving $\mathrm{N}_{2}$ injection and hence infer the geometry of the gas circulation 1035 plumes. The interpretation of these results with respect to existing literature on two-phase 1036 flow into granular media strongly support the hypothesis that the particle size distribution of 1037 the sand is an important factor controlling the geometry of gas circulation plumes, and hence 1038 of the resistivity anomalies obtained during the ERT monitoring. Sands with larger grain 1039 diameter $(d>500 \mu m)$ will tend to produce u-shape or parabolic anomalies, whereas in 
1040 smaller grain distributions $(d<400 \mu m)$, gas can accumulate within horizontal chambers

1041 which will produce flattened anomalies.

1042 Finally, we developed new acquisition protocols and produced a variety of tests to

1043 study their characteristics and performances. These investigations demonstrated that the

1044 capacity of one acquisition protocol to retrieve the true resistivity distribution after data

1045 inversion can vary significantly depending on the shape of the resistivity anomaly that is to be

1046 detected. Therefore, in order to optimize the measuring arrays for the monitoring of $\mathrm{CO}_{2}$ or

1047 any other gas transfer through the subsurface, one must carefully assess the prospective

1048 geometry of the gas circulation plume, and hence must gather relevant information on the

1049 geological structures composing the subsurface.

1050

1051

\section{ACKOWLEDGEMENTS}

1052

We gratefully acknowledge the support of the $\mathrm{CO}_{2}$ research program co-funded by ADEME, TOTAL and SCHLUMBERGER and their permission to publish this paper, and

1054 duely note that the views expressed here are those of the authors. We also thank Dr. Preignitz,

1055 Dr G. Donno, as well as an anonymous reviewer, whose comments greatly help to improve 1056 this article.

1057

1058

1059

1060

1061

1062

1063

1064 


\section{REFERENCES}

1074

1075

1076

1077

1078

1079

1080

1081

1082

1083

1084

1085

1086

1087

1088

1089

1090

1091

1092

1093

1094

1095

1096

1097

1098

1099

1100

1101

1102

1103

1104

1105

1106

1107

1108

Archie, G. E. (1942). The electrical resistivity $\log$ as an aid in determining some reservoir characteristics. SPE reprint series, 9-18.

Auken, E., Doetsch, J., Fiandaca, G., Christiansen, A. V., Gazoty, A., Cahill, A. G., \& Jakobsen, R. (2014). Imaging subsurface migration of dissolved CO 2 in a shallow aquifer using 3-D time-lapse electrical resistivity tomography. Journal of Applied Geophysics, 101, 31-41.

Baker, D. M. and Benson, C. H. (1996). Review of factors affecting in situ air sparging. In NonAqueous Phase Liquids (NAPLs) in Subsurface Environmental Assessment and Remediation, pages 292-310. ASCE.

Barrio, M., Bakk, A., Grimstad, A. A., Querendez, E., Jones, D. G., Kuras, O., ... \& Baudin, E. (2014). CO 2 Migration Monitoring Methodology in the Shallow Subsurface: Lessons Learned from the CO 2 FIELDLAB Project. Energy Procedia, 51, 65-74.

Bergmann, P., Schmidt-Hattenberger, C., Labitzke, T., Wagner, F. M., Just, A., Flechsig, C., \& Rippe, D. (2017). Fluid injection monitoring using electrical resistivity tomography-five years of CO2 injection at Ketzin, Germany. Geophysical Prospecting, 65(3), 859-875.

Bing, Z., \& Greenhalgh, S. A. (2000). Cross-hole resistivity tomography using different electrode configurations. Geophysical prospecting, 48(5), 887-912.

Binley, A., Ramirez, A. and Daily, W. (1995). Regularised Image Reconstruction of Noisy Electrical resistance Tomography Data, In: Process Tomography, by Beck, M.S. et al. (Eds.), Proc. Fourth Workshop of the European Concerted Action on Process Tomography, Bergen, Norway, pp. 401-410.

Binley, A., Siobhan H. and Shaw B. (1996a). Examination of solute transport in an undisturbed soil column using electrical resistance tomography. Water Resources Research. 32 (4), 763-769.

Binley, A., Shaw, B. and Siobhan, H. (1996b). Flow pathways in porous media: electrical resistance tomography and dye staining image verification. Measurement Science and Technology. 7 (3), 384390.

Binley, A. and Kemna, A. (2005). DC resistivity and induced polarization methods. Hydrogeophysics. Y. Rubin and S.S. Hubbard (ed.). Springer, Dordrecht, the Netherlands. p. 129-156. 
Böhler, U., Brauns, J., Hötzl, H., \& Nahold, M. (1990). Air injection and soil air extraction as a combined method for cleaning contaminated sites — observations from test sites in sediments and solid rocks. In Contaminated Soil. Vol 90 . (pp. 1039-1044). Springer Netherlands.

Bosch, D., Ledo, J., Queralt, P., Bellmunt, F., Luquot, L., \& Gouze, P. (2016). Core-scale electrical resistivity tomography (ERT) monitoring of CO 2-brine mixture in Fontainebleau sandstone. Journal of Applied Geophysics, 130, 23-36.

Breen, S.J., Carrigan, C.R., LaBrecque, D.J., Detwiler, R.L., (2012). Bench-scale experiments to evaluate electrical resistivity tomography as a monitoring tool for geologic $\mathrm{CO} 2$ sequestration. Int. J. Greenhouse Gas Control 9, 484-494.

Brooks, W, W., and M, A. (1996). Two phase flow patterns in porous media: An investigation relating to in-situ air sparging. In Proc. of the First Intl. Sym. on In Situ Air Sparging for Site Remediation.

Brooks, M. C., Wise, W. R., and Annable, M. D. (1999). Fundamental changes in situ air sparging patterns. Groundwater Monitoring \& Remediation, 19(2),105-113.

Busch, A., Alles, S., Krooss, B. M., Stanjek, H., \& Dewhurst, D. (2009). Effects of physical sorption and chemical reactions of CO 2 in shaly caprocks. Energy Procedia, 1(1), 3229-3235.

Carrigan, C.R., Yang, X., LaBrecque, D.J., Larsen, D., Freeman, D., Ramirez, A.L., Hovorka, S., (2013). Electrical resistance tomographic monitoring of CO2 movement in deep geologic reservoirs. Int. J. Greenhouse Gas Control 18, 401-408.

Chen, M.-R., Hinkley, R. E., and Killough, J. E. (1996). Computed tomography imaging of air sparging in porous media. Water resources research, 32(10), 3013_3024.

Christensen, N. B., Sherlock, D., \& Dodds, K. (2006). Monitoring CO2 injection with cross-hole electrical resistivity tomography. Exploration Geophysics, 37(1), 44-49.

Clayton, W. S. (1998). A field and laboratory investigation of air fingering during air sparging. Groundwater Monitoring \& Remediation, 18(3), 134-145.

Cox, P.M., Betts, R.A., Jones, C.D., Spall, S.A., Totterdell, I.J., (2000). Acceleration of global warming due to carbon-cycle feedbacks in a coupled climate model. Nature 408, 184-187.

Commer, M., Doetsch, J., Dafflon, B., Wu, Y., Daley, T. M., \& Hubbard, S. S. (2016). Time-lapse 3$\mathrm{D}$ electrical resistance tomography inversion for crosswell monitoring of dissolved and supercritical CO 2 flow at two field sites: Escatawpa and Cranfield, Mississippi, USA. International Journal of Greenhouse Gas Control, 49, 297-311.

Dahlin, T., \& Zhou, B. (2004). A numerical comparison of 2D resistivity imaging with 10 electrode arrays. Geophysical prospecting, 52(5), 379-398.

Daily, W., Ramirez, A., Binley, A. and LaBrecque, D. (2004). Electrical resistance tomography. The Leading Edge. 23 (5), 438-442.

Doney, S.C., Fabry, V.J., Feely, R.A., Kleypas, J.A., (2009). Ocean acidification: the other CO2 problem. Annu. Rev. Mar. Sci. 1, 169-192. 
Elder, C. R., \& Benson, C. H. (1999). Air channel formation, size, spacing, and tortuosity during air sparging. Groundwater Monitoring \& Remediation, 19(3), 171-181.

Glover, P. W. (2010). A generalized Archie's law for $n$ phases. Geophysics, 75(6), E247-E265.

Hermans, T., Wildemeersch, S., Jamin, P., Orban, P., Brouyère, S., Dassargues, A., \& Nguyen, F. (2015). Quantitative temperature monitoring of a heat tracing experiment using cross-borehole ERT. Geothermics, 53, 14-26.

Ji, W., Dahmani, A., Ahlfeld, D. P., Lin, J. D., and Hill, E. (1993). Laboratory study of air sparging : Air flow visualization. Groundwater Monitoring \& Remediation, 13(4): 115-126.

Johnson, R. L., Johnson, P., McWhorter, D., Hinchee, R., and Goodman, I. (1993). An overview of in situ air sparging. Groundwater Monitoring \& Remediation, 13(4): 127-135.

Keeling, R.F., Piper, S.C., Bollenbacher, A.F., Walker, J.S., (2009). Atmospheric CO2 records from sites in the SIO air sampling network. In: Trends: A Compendium of Data on Global Change. Carbon Dioxide Information Analysis Center, Oak Ridge National Laboratory, U.S. Department of Energy, Oak Ridge, Tenn., U.S.A.

Kiessling, D., Schmidt-Hattenberger, C., Schuett, H., Schilling, F., Krueger, K., Schoebel, B., and CO2SINK Group, (2010). Geoelectrical methods for monitoring geological CO2 storage: first results from cross-hole and surface-downhole measurements from the $\mathrm{CO} 2$ SINK test site at Ketzin (Germany). Int. J. Greenhouse Gas Control 4, 816-826.

Kremer, T., Schmutz, M., Maineult, A., and Agrinier, P., (2016a). Laboratory monitoring of $\mathrm{CO} 2$ injection in saturated silica and carbonate sands using spectral induced polarization. Geophysical Journal International. 2016a; 207 (2): 1258-1272. doi: 10.1093/gji/ggw333

Manabe, S., \& Stouffer, R. J. (1980). Sensitivity of a global climate model to an increase of CO2 concentration in the atmosphere. Journal of Geophysical Research: Oceans, 85(C10), 5529-5554.

Miller, C. R., Routh, P. S., Brosten, T. R., \& McNamara, J. P. (2008). Application of time-lapse ERT imaging to watershed characterization. Geophysics, 73(3), G7-G17.

Nakatsuka, Y., Xue, Z., Garcia, H., Matsuoka, T., (2010). Experimental study on CO2 monitoring and quantification of stored $\mathrm{CO} 2$ in saline formations using resistivity measurements. Int. J. Greenhouse Gas Control 4, 209-216.

Neftel, A., Moor, E., Oeschger, H., Stauffer, B., (1985). Evidence from polar ice cores for the increase in atmospheric CO2 in the past two centuries. Nature 315, 45-47.

Parasnis, D.S., 1997. Principles of Applied Geophysics. Chapmall and Hall.

Peter, A., Lamert, H., Beyer, M., Hornbruch, G., Heinrich, B., Schulz, A., ... \& Vogt, C. (2012). Investigation of the geochemical impact of $\mathrm{CO} 2$ on shallow groundwater: design and implementation of a CO2 injection test in Northeast Germany. Environmental Earth Sciences, 67(2), 335-349.

Peterson, J., Murray, K., Tulu, Y., Peuler, B., and Wilkens, D. (2001). Air-flow geometry in air sparging of fine-grained sands. Hydrogeology Journal, 9(2) :168_176. 
Pezard, P. A., Denchik, N., Lofi, J., Perroud, H., Henry, G., Neyens, D., ... \& Levannier, A. (2016). Time-lapse downhole electrical resistivity monitoring of subsurface $\mathrm{CO} 2$ storage at the Maguelone shallow experimental site (Languedoc, France). International Journal of Greenhouse Gas Control, 48, 142-154.

Priegnitz, M., Thaler, J., Spangenberg, E., Rücker, C., \& Schicks, J. M. (2013). A cylindrical electrical resistivity tomography array for three-dimensional monitoring of hydrate formation and dissociation. Review of Scientific Instruments, 84(10), 104502.

1226

1227

1228

1229

1230

1231

1232

1233

1234

1235

1236

1237

1238

1239

1240

1241

1242

1243

1244

1245

1246

1247

1248

1249

1250

1251

1252

1253

1254

1255

1256

1257

1258

1259

1260

1261

1262

1263

1264

1265

1266

1267

1268

1269

1270

1271

Raistrick, M., Hutcheon, I., Shevalier, M., Nightingale, M., Johnson, G., Taylor, S. \& Gunter, B. (2009). Carbon dioxide-water-silicate mineral reactions enhance $\mathrm{CO}_{2}$ storage; evidence from produced fluid measurements and geochemical modeling at the IEA Weyburn-Midale Project. Energy Procedia,1(1), 3149-3155.

Rücker, C., \& Günther, T. (2011). The simulation of finite ERT electrodes using the complete electrode model. Geophysics, 76(4), F227-F238.

Sellers, K. L. and Schreiber, R. P. (1992). Air sparging model for predicting groundwater cleanup rate. Proc. Petroleum Hydrocarbons and Organic Chemicals in Groundwater : Prevention,Detection, and Restoration.

Selker, J. S., Niemet, M., Mcduffie, N. G., Gorelick, S. M., \& Parlange, J. Y. (2007). The local geometry of gas injection into saturated homogeneous porous media. Transport in porous media, 68(1), 107-127. doi:10.1007/s11242-006-0005-0

Semer, R., Adams, J., and Reddy, K. (1998). TECHNICAL NOTE an experimental investigation of air flow patterns in saturated soils during air sparging. Geotechnical \& Geological Engineering, 16(1) :59_75.

Showstack, R., (2013). Carbon dioxide tops 400 ppm at Mauna Loa, Hawaii. Eos Trans. AGU 94, 192.

Szalai, S., Novák, A., \& Szarka, L. (2009). Depth of investigation and vertical resolution of surface geoelectric arrays. Journal of Environmental \& Engineering Geophysics, 14(1), 15-23.

Vialle, S., Contraires, S., Zinzsner, B., Clavaud, J.-B., Mahiouz, K., Zuddas, P., and Zamora, M. (2014). Percolation of CO2 rich fluids in a limestone sample : Evolution of hydraulic, electrical, chemical, and structural properties. Journal of Geophysical Research : Solid Earth, 119(4) : 2828_2847.

Wehrle, K. (1990). In-situ cleaning of CHC contaminated sites : model-scale experiments using the air injection (in-situ stripping) method in granular soils. In Contaminated Soil 90, 1061-1062. Springer.

Xu, T., Apps, J. A., \& Pruess, K. (2005). Mineral sequestration of carbon dioxide in a sandstone-shale system. Chemical geology, 217(3), 295-318.

Xue, Z., Tanase, D., Watanabe, J., (2006). Estimation of CO2 saturation from time-lapse CO2 well logging in an onshore aquifer, Nagaoka, Japan. Explor. Geophys. 37, 19-29.

Yang, X., Lassen, R. N., Jensen, K. H., \& Looms, M. C. (2015). Monitoring CO 2 migration in a shallow sand aquifer using 3D crosshole electrical resistivity tomography. International Journal of Greenhouse Gas Control, 42, 534-544. 
1272

1273 